\title{
Jens Peter Laut
}

Errata et Corrigenda in alttürkischen Handschriften in
sogdo-uigurischer Schrift 
Jens Peter Laut

\section{Errata et Corrigenda in alttürkischen Handschriften in sogdo-uigurischer Schrift*}

\section{Vorbemerkung}

Die „Handschriftenkunde“ des Alttürkischen, d. h. die Erforschung der äußeren Gestaltung der Manuskripte von der Paläographie bis zu den figurativen Ausschmückungen, steckt noch in den Anfängen. ${ }^{1}$ Es ist schon von daher besonders reizvoll, sich diesem Themenkreis zuzuwenden, und ich hoffe, anhand einiger Ergebnisse meiner Untersuchungen eines begrenzten Ausschnittes zeigen zu können, daß die Erforschung uigurischer Texte nach den Gesichtspunkten der Handschriftenkunde manche interessanten Resultate erbringen kann.

Die beiden neutürkischen Redensarten insan beșer kuldur șașar ,irren ist menschlich“ und bukadar kusur kadı kı̨ında da bulunur ",nichts ist ohne Fehler" gelten auch für die Schreiber der ehrwürdigen alttürkischen Manuskripte. Diese Handschriften weisen eine Fülle von Verschreibungen, Auslassungen und anderen Fehlern bzw. Besonderheiten auf, die von der Forschung - wenn überhaupt - bisher nur in kurzen Anmerkungen in den textkritischen Apparaten zur Kenntnis genommen worden sind. ${ }^{2}$ Die Frage, wie die uigurischen Schreiber mit diesen ihren handschriftlichen Fehlleistungen umgegangen sind, d. h. welche Möglichkeiten von Korrekturmaßnahmen ihnen zur Verfügung standen, ist bisher ebenfalls nicht weiter beachtet worden. Ich möchte im folgenden, ohne Anspruch auf Vollständigkeit, auf einige der typischen handschriftlichen Errata sowie auf das breitgefächerte System der Korrektur-Varianten von uigurischen Schreibern hinweisen. Es wird sich dabei u. a. auch zeigen, daß manche Bestandteile atü. Manuskripte nicht wie bisher als „Fehler“ bzw. deren „Korrekturen“ apostrophiert werden können.

\footnotetext{
* Leicht überarbeitete Fassung eines Vortrages, gehalten auf der 31. Tagung der Permanent International Altaistic Conference, Weimar, Juni 1988.

${ }^{1}$ Es ist bezeichnend, daß das einzige Werk, welches sich - beschränkt auf die Varianten der sogdo-uigurischen Schrift - ausführlicher mit alttürkischer Paläographie beschäftigt, bereits 1919 (!) erschienen ist: A. v. Le Coq, Kurze Einführung in die uigurische Schriftkunde, in: Mitteilungen des Seminars für Orientalische Sprachen an der Friedrich-Wilhelms-Universität zu Berlin. Westasiatische Studien. 22. 1919. 93-109. Nachdruck in: Sprachwissenschaftliche Ergebnisse der deutschen Turfan-Forschung. 3. Leipzig 1985, 519-537. - Paläographische Fragen zur manichäischen Schrift sind behandelt in P. Zieme, Untersuchungen zur Schrift und Sprache der manichäisch-türkischen Turfantexte. Berlin 1969 (Unpubl. Diss. phil.). In naher Zukunft wird OStR Dr. D. Maue (Gießen) die Ergebnisse seiner Untersuchungen der atü. Texte in Bräbmi-Schrift vorlegen, ebenfalls in Vorbereitung ist eine Studie von D. Fedakâr, M. A. (Gießen) zur Paläographie der atü. Fragmente in sogdischer Schrift. J. P. Laut wird, im Rahmen seiner geplanten Gesamtedition der MaitrisimitHandschriften aus Sängim, Murtuk und Hami, diese auch nach paläographischen Fragestellungen untersuchen.

${ }^{2}$ Eine kleine Auswahl von Schreibfehlern und anderen Versehen in den Hss. der Maitrisimit aus Hami und Sängim zur Herausarbeitung der jeweils besseren Lesart ist zusammengestellt und ausgewertet bei J. P. Laut: Der frühe türkische Buddhismus und seine literarischen Denkmäler. Wiesbaden 1986, 50-54. (Veröffentlichungen der Societas Uralo-Altaica. 21.)
} 
Zu erwähnen bleibt, daß ich bei allen folgenden Textbeispielen die „Gießener Umschrift" und, aus rein praktischen Gründen, waagerechte Leserichtung zugrundelege.

\section{Die Sängimer Handschrift der Maitrisimit}

Beginnen wir mit den wirklichen Errata, wobei es sinnvoll erscheint, das frühe (ca. 9./ 10. Jh.) Sängimer Manuskript ${ }^{3}$ der Maitrisimit (im folgenden: MaitrSängim) an den Anfang zu stellen. Die sicherlich noch ältere „Londoner Rolle“ des Säkiz Yükmäk YarokSütra kann in unserem Zusammenhang nicht herangezogen werden, da sie - und dies spricht für die Qualität des Schreibers - keine nennenswerten Fehler und Korrekturen aufweist. ${ }^{5}$

Auch MaitrSängim ist eine - gerade im Vergleich mit vielen jungen Manuskripten recht sorgfältig geschriebene Handschrift, was eine erhebliche Anzahl „Errata et Corrigenda" jedoch nicht ausschließt. Für uns unerheblich sind dabei die in praktisch allen uigurischen Manuskripten erscheinenden kleinen „Flüchtigkeitsfehler“" ${ }^{\text {, }}$, etwa das häufige Vergessen eines L-Hakens oder von Affixen (+ka, +nı $\eta$ usw.): letztere werden zumeist in kleinerer Schrift dem dazugehörigen Wort superskribiert.

Betrachten wir im folgenden aber einige der für MaitrSängim charakteristischen Fehler und Korrekturen:

\section{1 ,Auslassungszeichen“:}

Kennzeichnend in MaitrSängim ist hierfür ein kleines Kreuz (+), welches unter die Zeile an diejenige Stelle gesetzt wird, wo ein Wort oder deren mehrere vergessen worden sind. Die fehlenden Bestandteile werden dann der Stelle superskribiert (Abb. 1) ${ }^{-}$. Das Kreuzchen ist das einzige gebräuchliche Korrekturzeichen in MaitrSängim, und dies unterscheidet die Handschrift deutlich von späteren Manuskripten, in denen mebrere Korrekturzeichen mit verschiedenen Funktionen bezeugt sind (s. u.). Ich möchte hieraus schließen, $\mathrm{da}$ in früherer Zeit die Verwendung von solchen Zeichen und hierzu zähle ich auch

\footnotetext{
${ }^{3}$ Vgl. Laut, Der frühe türkısche Buddhısmus (s. Anm. 2), bes. $59 \mathrm{ff}$.

${ }^{4} \mathrm{Vgl}$. op. cit., bes. $78 \mathrm{ff}$.

"Hingewiesen sei lediglich auf Z. 227, wo falsches olur ,stirbt“ gestrichen und togar ,wird geboren“ superskr1biert ist, sowie auf Z. 228, die nachträglich und mit dünnerem Kalem zwischen die /e1len 227 und 229 eingefügt ist. Ein Faksimile der „Londoner Rolle“, lange Zeit ein Desiderat, 1st jetzt von J. Hamılton veröffentlicht worden: Manuscrits Oü̈gours du IX ${ }^{\mathrm{c}}-\mathrm{X}^{\mathrm{e}}$ siècle de Touen-Houang. 2. Parss 1986, 331-350.

${ }^{6}$ Welche Folgen solche kleinen, oft vom Schreiber nicht bemerkten und nicht horrigierten Fehler fur spatere Editoren haben können, zeigt das Beispiel P'LyQ 'Q in Taf. 7 v. 16 (alle Angaben von Tafel [= Taf.] und Zeile [=Z.] nach: A. v. Gabain: Maitrisimit. Faksimile der alttürkischen Versıon eines Werkes der buddhistıschen Vaibhāṣika-Schule. [I]. Wiesbaden 1957. II. Berlin 1961). Șinasi Tekın hat in seiner Edıtıon des 'Textes $p$ 'ly $\ddot{q}{ }^{\prime} z$ (mit der Einschränkung: „Man könnte auch 'q lesen“) zugrundegelegt und als \omına propria Balïq und A $<$ interpretiert (Ș. Tekin: Maitrisimit nom bitig. Die uigurische Übersetzung eines Werkes der buddhistıschen Vaibhāșika-Schule. 1. Berlin 1980, 41. Berliner Turfantexte. 9. [Im folgenden: BT 9]). Hier 1st jedoch, wie die Parallelstelle der Hami-Handschrift der Maitrisimit 1. Kap., Blatt 1 v. 5 ceigt, lediglich ein L vergessen worden, und es ist in balık $\langle l\rangle a g$,städtisch“ zu emendieren (Zu den bisherigen Fditıonen der Hami-Hs. s. J. P. Laut: Bemerkungen zu den jüngsten Editionen der Hami-Handschrıft der Maitrısimit. In: Ural-Altaische Jahrbücher, N. F. 9 [1990], 257-265).

Z. B. Taf. 4 v. 28. Ich danke Herrn Dr. M. Kraatz (Religionskundliche Sammlung, Marburg) für seine freundliche Unterstützung bei den fotografischen Arbeiten.
} 
das einfache Durchstreichen von Worten nicht sehr beliebt war, und daß die Schreiber versuchten, ihre Fehler so unauffällig wie möglich zu korrigieren. Man schrieb ja schließlich oft im Auftrag reicher Stifter, die saubere Manuskripte sehen wollten. So wären auch die für MaitrSängim typischen ,,unauffälligen“ oder ,,versteckten“ Korrekturen zu erklären:

\section{2 „Versteckte Korrekturen“:}

Mit diesem Begriff möchte ich diejenigen Korrekturen bezeichnen, die innerbalb eines Wortes angefertigt werden, also nicht sub- oder superskribiert werden. In MaitrSängim sind folgende Möglichkeiten von Emendationen durch ,,versteckte Korrekturen“ zu konstatieren:

2.2.1 Durch Übermalen falscher Grapheme: So in Taf. 2 r. 2, wo ursprünglich TWYMK'ऽYZ/ tümgäsiz, ,nicht töricht" statt richtigem tümgäsi, ,ihr Törichtsein“ geschrieben wurde (Abb. 2). Der Schreiber nutzte die ansonsten nur am Zeilenende bestehende Möglichkeit der Verdoppelung des letzten Graphems (,Zeilenfülier") und malte ein y über das finale Graphem $z^{\sharp}$. Ähnlich sst der Fall in Taf. 134 r. 8, wo zunächst YwQ-Q’ geschrieben worden war. Der Schresber hatte hier die leichte Möglichkeit, durch Anfügung eines L-Hakens falsches yokka in richtiges yolka, auf den Weg“"zu emendieren (Abb. 3). Nur einem damaligen Kenner der sogdo-uigurischen Schrift - und wir können wohl davon ausgehen, daß die Stifter nicht zu dieser Elite gehörten - wird aufgefallen sein, daß der obere 'Teil dieses L etwas breit geraten ist. Im Falle eines zunächst versehentlich geschriebenen ölürmätin ('Taf. 131 r. 20) hatte der Schreiber die elegante Möglichkeit, das „fal-

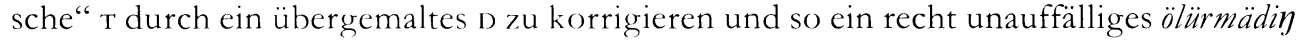
„du hast nicht getötet“ zu erhalten (Abb. 4). Weniger schön ist die Korrektur eines Schreibers der Höllenkapitel von MaitrSängim geraten, der (in Taf. 73 r. 25) ursprünglich an das Konverb ärksinip (<ärk,sin- ,Macht haben über etwas“) gedacht haben mag. Nach dem Schreiben des s muß er sich entschlossen haben, eine Emendation in äyänip (< <äクän,sich etwas anmaßen“) vorzunchmen und hat versucht, das s durch Alif zu übermalen. Daß dies von Form und Größe der beiden Grapheme her mißlingen mußte, liegt auf der Hand, und auch in der bislang letzten Edition wird das Wort falsch gelesen?"

2.2.2 Durch Übermalen korrekter Grapheme: So in Taf. 18 r. 14, wo das Graphem N in falschem DNT'R / $d(i)$ ntar ,Mönch“ durch Hinzufügung eines kleinen Linksbogens zu „Quasi-YN“ gemacht wurde (Abb. 5).

2.2.3 Durch Anfügung von Graphemen: So in Taf. 16 v. 10, wo der Schreiber zunächst känt ulušta für ,aus der Stadt " " geschrieben hatte. Ich vermute, daß hier, ebenso wie an einer anderen Stelle von MaitrSängim ${ }^{10}$ dem Schreiber der Gebrauch des Lokativ-Ablativ-Affixes + D' nicht mehr deutlich war, so daß er eine Emendation in das „moderne“

\footnotetext{
${ }^{8}$ Ich möchte diese Frklärung jetzt vorziehen; korr. Laut, Der frühe turkısche Buddhısmus (s. Anm. 2), 49, Anm. 2.

"BT 9, 185, Z. 25: "rksnyp. Tekin schreibt dazu: „Das Wort 1st verderbt. Meine Lesung nach dem Zusammen-

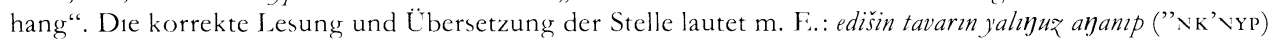
maniy ol išl (a)tdim „Gefaße und Habe (des Sañgha) maßte 1 ch fur mich allen an und benutzte sie, (1ndem ich dachte:),Dies 1st meins!"” (Taf. 73 r. 24-26).

1" Vgl. Laut, Der fruhe türkısche Buddhismus (s. Anm. 2), 62.
} 
Affix + DYN vornahm. Er konnte jedoch nicht mehr ein einfaches Übermalen des finalen Alif zu y vornehmen, da er den Horizontalstrich des ,falschen“ Alif bereits langgezogen hatte - und dies ist bei y nicht möglich. Er zog sich m. E. damit aus der Affäre, daß er dem finalen Alif ein Y anfügte und nach einem Spatium ein isoliertes N schrieb (Abb. 6). Dadurch wurde das Schriftbild nicht entscheidend gestört und zugleich war der der uigurischen Schrift Kundige in der Lage, die Emendation durch die Unregelmäßigkeit als solche zu erkennen.

2.3 Dieses letzte Beispiel führt uns etwas aus dem Bereich der ,normalen“ Fehlleistungen hinaus und soll in unserem Zusammenhang zeigen, daß auch morphologische und grammatische Corrigenda in MaitrSängim in Form von „versteckten Korrekturen“ vorgenommen wurden ${ }^{11}$. Hierzu gehören ja auch die Emendationen tınlag zu tınlıg (Taf. 64 r. 8) und bašlag zu bašlıg (Taf. 73 v. 20; Abb. 7), wo das Alif durch Hinzufügung eines kleinen Linksbogens zu y ,umfunktioniert“" wurde ${ }^{12}$.

2.4 Wir hatten bisher gesehen, daß die Schreiber von MaitrSängim bemüht waren, die von ihnen erkannten Fehler oder was sie dafür hielten, so unauffällig wie möglich zu korrigieren. Die Unauffälligkeit möchte ich, wie oben erwähnt, auf das Bestreben zurückführen, dem Auftraggeber ordentliche, und seien es auch nur scheinbar ordentliche, Manuskripte zu liefern. Dieser hätte trotz Unkenntnis der Schrift auffällige Korrekturen sehr wohl als solche erkannt, und wir können wohl davon ausgehen, daß in einem solchen Fall Honorarkürzungen erfolgten. Daß dies nicht in der Absicht der Schreiber lag, ist verständlich, ebenso jedoch auch ihr Bemühen, überhaupt Korrekturen anzubringen: a) aus Gründen des höheren religiösen Verdienstes und b) aus praktischen Zwecken, dienten diese Texte doch dem internen mönchischen Gebrauch und sollten auch von daher möglichst fehlerfrei sein. Daß dennoch mancher Fehler stehenblieb, ist eingangs erwähnt worden. Die meisten nicht-korrigierten Fehler in MaitrSängim betreffen, wie ich an anderer Stelle ausgeführt habe ${ }^{13}$, Wörter mit ähnlichem Schriftbild, z. B. sözlä-statt süzül-, išläti statt eštilti usw. In eine ähnliche Kategorie fällt die Verschreibung tört törlüg ,viererlei“ statt tört tümän „40.000“14, wo zur Ähnlichkeit des Schriftbildes auch die Stereotypie des überaus häufigen Ausdrucks tört törlüg kommt, was einen Schreiber sicher zu seiner Fehlleistung verführen kann. Fehler dieser Art zeigen m. E., daß die Schreiber zumindest in solchen Fällen nicht etwa nach Diktat eines Mitmönches geschrieben, sondern eine Abschrift eines ihnen vorliegenden Textes vorgenommen haben. Als weitere Hinweise auf eine Abschrift nach Augenschein und nicht nach Gehör möchte ich die recht häufigen Dittographien in MaitrSängim werten, seien es die von einzelnen Graphemen bzw. Graphemgruppen ${ }^{15}$, von kleineren Syntagmen (Abb. 8) ${ }^{16}$, oder von ganzen längeren Satzteilen (Abb. 9) ${ }^{17}$. Es ist schwer vorstellbar, daß zwei

${ }^{11}$ Im Falle der Taf. 5 r. 16 gab es für den Schreiber keine andere Möglichkelt, als das Wort barıp zu superskribieren (vgl. Laut, Der frühe türkische Buddhismus [s. Anm. 2], 62.).

${ }^{12} \mathrm{Vgl}$. loc. cit. Die Hinzufügung eines y-Bogens konnte also sowohl ein Graphem zu y ändern, als auch, wie oben bei 2.2.2 gezeigt, das betreffende Graphem um Y erweitern.

${ }^{13} \mathrm{Vgl}$. op. cit., 49-54.

${ }^{14}$ Vgl. op. cit., 52.

${ }^{15}$ Z. B. Taf. 29 r. 5: ärdni\{ni\}lig.

${ }^{16}$ So z. B. yüz yašlag bolur in Taf. 122 v. 11-12.

${ }^{17}$ So in Taf. 167 r. 13-23 (BT 9, 152; normalisierte Transkription, ohne Angabe von Zerstörungen): (13) siz̨lärni osuglug mänilig (14) tonalıg uzun yašlıg tınlgglar bolur- (15) lar . ol antag sansar ičinta tenlıg (16) yok kim ol 
Mönchen, also dem Vorleser und dem Schreiber eines Textes, gleichzeitig Fehler dieser Art unterlaufen sollten.

2.5 Weitere Fehlergruppen sind graphisch komplizierte türkische Wörter und Lehnwörter, so etwa 'M'RLR'RY (Taf. 173 r. 1) für amarlları oder PR'TYK'NR'R'K (Taf. 176 v. 27) für pratikanarak u. ä. (< sgd. *prtykn(") rk < skr. pratyekanaraka ,Spezialhölle“) ${ }^{18}$. Sind die beiden zuletzt erwähnten Fälle ein Zeugnis für eher triviale Flüchtigkeitsfehler, so

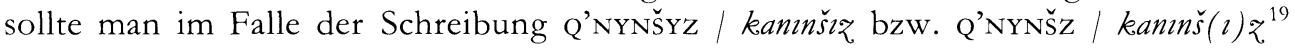
anstelle von Q'NYNČsYZ / Kanınčsız, „unersättlich“ nicht von einem „Fehler“ des Schreibers ausgehen ${ }^{20}$. Wie die häufigen Belege nahelegen, handelt es sich eher um eine kontaminierte Form $(\check{c}+s \rightarrow \breve{s})$, die auf dialektale Einflüsse zurückgeführt werden könnte.

2.6 Nun gibt es auch in MaitrSängim einige superskribierte Wörter, die jedoch zum allergrößten Teil auf spätere Leser der Handschrift zurückgehen, die sich bemüßigt fühlten, Korrekturen anzubringen. Sehr sinnvoll ist dies im Falle von bereits in alter Zeit verblaßten oder verwischten Bestandteilen des Manuskriptes. So ist in Taf. 15 r. 6 über verblaßtes munta kim mit Pinselkursive munda kim nachgetragen worden. Das $\mathrm{N}$ ist bezeichnenderweise - wie in späterer Zeit üblich - punktiert, ebenso wie zwei Zeilen vorher, wo auf unleserliches braman taク mit dem Pinsel diese Worte nachgezogen sind.

\section{Das „Uigurische Totenbuch“ und andere späte Handschriften}

Betrachten wir im Gegensatz zur sorgfältig geschriebenen frühen Handschrift MaitrSängim (ca. 9./10. Jh.) zunächst einen der großen Textzeugen der Spätzeit, das „Uigurische Totenbuch" aus dem 14. Jahrhundert (im folgenden: UigTot) ${ }^{21}$. Es fällt sofort auf, mit welcher Nachlässigkeit dieses Manuskript geschrieben wurde, und es ist kaum vorzustellen, daß Handschriften dieser Art - und hierzu zählen auch die unten zu besprechenden Avadāna-Texte - noch im Auftrage von Stiftern angefertigt wurden: Sie werden nur für mönchische Zwecke, und das heißt für eigenes Studium und Predigten vor Laien bestimmt gewesen sein. Dies soll später anhand von bestimmten Besonderheiten ausführlicher gezeigt werden.

Je mehr verschiedene Fehler gemacht werden, desto mehr Korrekturzeichen sind nötig, wie am Beispiel des „Totenbuches“ ersichtlich:

3.1 Stellen, an denen ein Wort bzw. ein Affix nachgetragen werden muß, sind mit einem

irinč tınlıglar osuglug (17) bolmasarlar antag ymà tınlıg yok (18) kim sizlärni osuglug mäyilig tojalıg (19) u₹un yašlıg tınlıglar bolurlar . ol (20) antag sansar ičintä tınlıg yok. kim (21) ol irinč tınlıglar osuglug bolmasar- (22) lar . antagymä tınlıg yok kim sizlär- (23) ni osuglug mäyilig tojalıg bolmasar. Der Schreiber hat die Zeilen nicht, wie Tekin meint, „weil ein grammatischer Fehler gemacht worden war" (BT 9, 152, Anm. 113) wiederholt, sondern weil er bei der Abschrift von Z. 18 versehentlich wieder mit dem Blick in Z. 14 gekommen sein muß. Sein nochmaliges Schreiben des ganzen Passus kann ebenfalls der Kategorie „Versteckte Korrekturen“ zugeordnet werden.

${ }^{18} \mathrm{Vgl}$. Laut, Der frühe türkische Buddhismus (s. Anm. 2), 103-104, Anm. 7.

${ }^{19}$ Taf. 55 v. 10 ; Taf. 157 v. 25, 31; Taf. 196 r. 13.

${ }^{20}$ So in BT 9, 134, Anm. 103, 104; 156, Anm. 114; 245, Anm. 173.

${ }^{21}$ Peter Zieme und György Kara: Ein uigurisches Totenbuch. Nāropas Lehre in uigurischer Übersetzung von vier tibetischen Traktaten nach der Sammelhandschrift aus Dunhuang British Museum Or. 8212 (109). Wiesbaden 1979. (Asiatische Forschungen. 63.) 
Kreuzchen (+) markiert und das nachzutragende Wort (Abb. 10) bzw. Affix (Abb. 11) ist der Stelle superskribiert ${ }^{22}$.

3.2 Stellen, an denen ein Graphem nachgetragen werden muß, sind durch einen kleinen Kreis markiert und das Graphem ist der Stelle superskribiert (Abb. 11) ${ }^{23}$.

\subsection{Zu emendierende Wörter sind:}

3.3.1 Mit einem T-förmigen Zeichen verschiedener Größe unterlegt, und das richtige Wort ist der Fehlschreibung superskribiert (Abb. 10) ${ }^{24}$,

3.3.2 Durch einen langen Unterstrich getilgt, und das richtige Wort ist der Fehlschreibung superskribiert ${ }^{25}$.

3.4 Das einfache Durchstreichen und Unleserlich-Machen eines Wortes war im „Totenbuch" nicht üblich: Ist das ein weiterer Hinweis darauf, daß das einmal Geschriebene in religiösen Texten als „,sakrosankt“ angesehen wurde? Dies heißt jedoch nicht, daß die Uiguren diese Korrektur-Methode nicht auch anwendeten: So z. B. im Fragment Mainz $713^{26}$, wo in Z. 45 das Wort täg „wie“ gestrichen und durch + ka obšatı ,vergleichbar" ersetzt wurde (Abb. 16) ${ }^{27}$. In den yüanzeitlichen Ligurica, die Șinasi Tekin ediert hat ${ }^{28}$, ist das einfache Durchstreichen falscher Wörter mit Superskribierung sogar die häufigste Korrekturmaßnahme bei Emendationen ${ }^{29}$. Erwähnenswert ist im zweiten Teil des Werkes das Umrahmen eines zu emendierenden Wortes (Abb. 18) ${ }^{3(1)}$. Eine ähnliche und

${ }^{22}$ Vgl. LigTot, Z. 918 (Taf. 42 b, S. 325, [Abb. 10]), wo nach/utragendes tıdıgin uber der \%elle steht bLw. UigTot, Z. 239 (Taf. 11 b, S. 294, [Abb. 11]), wo das Affix + ka superskribiert kst: Beide Male markiert ein Kreuzchen etwas unter der Zeile die Stelle des Nachtrags.

${ }^{23}$ Vgl. Lig'Tot, Z. 241 (Taf. 11 b, S. 294), wo nach/utragendes I von tar $\langle t\rangle$ dac̆ uber der \%erle steht und ein kleiner Kreis unter der Zeile auf die Stelle hinweist.

${ }^{24}$ Vgl. UigTot, 7. 918 (Taf. 42 b, S. 325), wo /u emendierendem turkaru das T-/.eichen subskribiert ist und richtiges torku „Se1de“ superskribiert ist. In \%. 491 (Taf. 23 a, S. 3(15) 1st das Lu tilgende ootun mit einem sehr großen T-Zeichen unterlegt, welches fast das gesamte W ort umfaßt: Richtigcs ootin ist supcrskribiert (Abb. 12). Der Schreiber hatte anscheinend seine Schwierigkeiten mit dem If ort o,t „Feuer"; so liegen neben der eben genannten Stelle noch zwer Korrekturen in \%. 43 (Taf. 2 b, S. 285, Abb. 13) und \%. 176 ('Taf. 8 b, S. 291; Abb. 14) vor: Hier sind die Fehlschreibungen od bzw. ood mit dem T-/eichen unterlegt und mit richtigem oot superskribiert. Fehler dieser Art können also Hinwerse darauf bieten, ob es sıch um denselben Schrerber handelt.

${ }^{25} \mathrm{Vgl}$. UigTot, Z. 120 (Taf. 6 a, S. 288; Abb. 15), wo falsches ațasina auf diese Art durch korrektes atasinı ersetzt wurde, oder 7. 129, wo ebenso bulur in bilur emendiert wurde.

${ }^{26} \mathrm{Vgl}$. J. P. Laut und P. Zieme: Ein zweisprachiger Lobpreis auf den Bag von Kočo und seine Gemahlın. In: J. P. Laut und K. Röhrborn (Hrsgg.): Buddhistische Frzahlliteratur und Hag1ographie in turk1scher Überlieferung. Wiesbaden 1990, 15-36. (Veröffentlichungen der Socretas Uralo-Altaica. 27.)

$2^{-}$Es ist unklar, ob diese Änderung metrı causa oder aus stilistischen Gründen vorgenommen wurde.

${ }^{28}$ Ș. Tekin: Buddhistische Uigurica aus der Yüan-Zeit. Teil I: HSIN Tözın Oqıdtači Nom. Teıl II: Die Geschichte von Sadāprarudita und Dharmodgata Bodhisattva. Budapest 1980. (Bibliotheca Orientalıs Hungarica. 27.)

29) Vgl. z. B. Teil II, Z. 385 (Taf. 30). Das einfache Durchstreichen zu emendierender Wörter, eine Korrekturmaßnahme der uig. Spätzeit, ist insbesondere in sehr nachlässigen Mss. bezeugt. Dem durchgestrichenen W'ort kann die Emendation auch subskribiert sein, vgl. etwa Hamilton, Manuscrits (s. Anm. 5), T. 1, 78, Z. 15 (Faks. in op. cit., T. 2, 291 ['Text 14]), wo getilgtem tort „4“ korrektes $i k i, 2$ “" subskribiert 1st (Abb. 17).

") Tekin, op. cit., Te1l II, Z. 103 (Taf. 22), wo falsches oru kodı ellipsenförmig umrahmt ist und das korrekte oydun kidin superskribiert ist. 
ebenso seltene Emendation durch eine gestrichelte Umrahmung findet sich im uig. Sukhävatīvy ūha ${ }^{31}$.

3.5 Ich hatte oben darauf hingewiesen, daß im „Uigurischen Totenbuch“ ein kleiner Kreis unter dem Wort dazu dient, die Stelle eines fehlenden Graphems zu markieren, welches dem Wort superskribiert ist (vgl. Abb.11 und Anm. 23). Der gleichen Korrekturmaßnahme wie in diesem nachlässigen Manuskript des 14. Jahrhunderts bedienten sich die Schreiber der lange Zeit für einen Blockdruck gehaltenen Prachthandschrift des uigurischen Goldglanz-Sütra aus dem 17. Jahrhundert, wie zwei in Faksimile veröffentlichte Blätter zeigen ${ }^{32}$. Hier werden jedoch nicht nur die Stellen für vergessene Grapheme ${ }^{33}$, sondern auch die für ganze Wörter mit einem kleinen Kreis markiert ${ }^{34}$. In jedem Fall hat das Korrekturzeichen ,,kleiner Kreis“ in diesen späten Handschriften die Funktion, die Stellen für nachträglich einzufügende Textbestandteile zu markieren.

\section{Späte liadāna-Texte}

Eine bemerkenswerte Ausnahme scheinen mir hier die von M. Shōgaito edierten Avadāna-Texte zu bilden, die jüngst in einem Sammelband neubearbeitet herausgegeben wurden ${ }^{35}$.

4.1 In diesen Texten markiert ein kleines Kreuz die Stellen, an denen der Zeile superskribierte Bestandteile einzufügen sind ${ }^{36}$. Den zu tilgenden Wörtern ist, wie auch im „Uigurischen Totenbuch", cin 'T-förmiges Zeichen subskribiert und die Emendationen stehen über der Zeile ${ }^{3-}$.

4.2 Welche Funktion ist nun dem kleinen Kreis zugedacht, der in unseren Avadanas häufig erscheint? Er ist in diesen Texten unter bestimmte Wörter gemalt, denen wiederum andere Wörter superskribiert sind. Auf den ersten Blick scheint es, als läge eine spielerische Variante des ,normalen“ T-förmigen Tilgungszeichens vor. Dies nimmt

${ }^{31}$ P. Zieme: U1gurische Sukhäratīi üha-Fragmente. In: Altorientalische Forschungen 12 (1985), 129-149. Hier 1st, 1n Z. 67 (S. 143, dazu Taf. VII v. 2) zu emendierendes 'wR durch Lmstrichelung getilgt und korrektes yer dahintergeschrieben.

${ }^{32}$ K. Kudara und K. Rohrborn: Zwe verırrte Blatter des uigurıschen Goldglanz-Sütras im Etnografiska Museum, Stockholm. In: ZDMG 132 (1982), 336-347.

${ }^{33}$ Z. 8 (Y), Z. 27, 42 (Alif). Fine kleine Besonderhe1t liegt in Z. 70 vor, wo das vergessene Suffix $+i n$ dem zugehörıgen Wort ugur subskribiert und nıcht wie ublich superskribiert ist.

${ }^{34}$ Z. 25 (kamag). Im „Totenbuch“ wird fur diesen Fall hingegen ein Kreuzchen benutzt (vgl. Anm. 22).

${ }^{35}$ M. Shōgaito: Drei zum 1l'alokitésvara-sūtra passende Avadānas. In: J. P. Laut und K. Röhrborn (Hrsgg.): Der türkische Buddhismus in der japanıschen Forschung. Wiesbaden 1988, 56-99 [Fakss.: 107-119]. (Veröffentlichungen der Societas Lralo-Altaica. 23.)

${ }^{36}$ Vgl. z. B. Z. 41 (Abb. 19), wo oru ,aufwärts, hoch“ oder Z. 164 (Abb. 20), wo (chin.) te ien ṫ ien [= uig. tayri taクri] (uig.) si burbanka einzufügen sind.

${ }^{37}$ Vgl.z. B. Z. 164 (Abb. 20): y'ma fur antag; Z. 202 (Abb. 21): ayančayligın uduzup balık.ka kigürdilar , sie führten ehrfürchtig und geleiteten in die Stadt“ für čayška ötüntilär ,sıe luden zum Essen ein“. Im letzten Beispiel sind

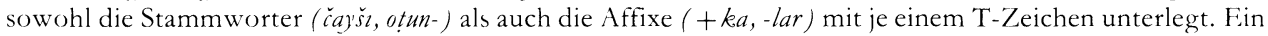
kleines Kurıosum 1st ein getılgter „Funfpunkt“ ( $\therefore$ ) in Z. 8, der durch Einkreisen der äußeren 4 Punkte zum „Einpunkt“ umgewandelt wurde, welcher nun korrekt das Versende und nicht, wie ein Fünfpunkt, das Strophenende anzeigt. 
auch der Bearbeiter der Texte, M. Shōgaito, an, wie aus seinen Anmerkungen zu den im folgenden genannten Textzeilen ersichtlich ist ${ }^{38}$. Betrachten wir einige der Belege genauer (an erster Stelle steht das durch einen oder mehrere kleine Kreise subskribierte Wort, nach dem Doppelpunkt das superskribierte Wort):

Z. 54 (Abb. 22): adaka: ämgäkkä; Z. 115 (Abb. 23): tägintilär: täginip; Z. 165 (Abb. 24): burban: (chin.) fo; Z. 251 (Abb. 25): taฤısuk: säcmä; Z. 275 (Abb. 26): küülüg: yü̈rlüg.

Es scheint mir ohne Zweifel, daß hier keine Emendationen, sondern alternative Lesarten vorliegen, d. h. der kleine Kreis will ein betreffendes Wort nicht tilgen, sondern weist auf eine über diesemWort stehende semantisch-stilistische Variante hin. In den

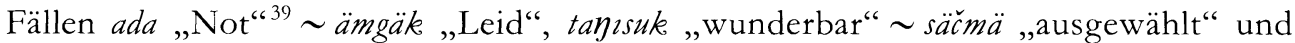

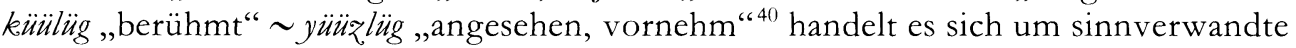
Wörter, die je nach Intention austauschbar sind.

Das Beispiel burban „Buddha“ (chin.) fo „Buddha“ zeigt durch ebendiese Gegenüberstellung ganz deutlich, daß einige chinesische Wörter in diesen alttürkischen Texten der Spätzeit gewissermaßen zum „,buddhistischen Grundwortschatz“ “41 gehörten und beliebig austauschbar mit ihren uigurischen Äquivalenten waren.

Eine syntaktische Variante liegt im Falle tägintilär ,,sie näherten sich“ täginip (Konverb) $\operatorname{vor}^{42}$.

4.3 Das Vorhandensein von alternativen Lesarten läßt m. E. zwei Dinge als wahrscheinlich gelten: 1. Die betreffenden Texte sind zumeist ad hoc komponierte Predigten, die keinen direkten Ausgangstext haben und deren Schreiber zugleich ihr Verfasser gewesen sein wird. 2. Die Texte waren zum mündlichen Vortrag, wohl zur Erbauung von Laien, bestimmt. Diese beiden Vermutungen werden auch durch inhaltliche Passagen der Avadānas bekräftigt, die die zeitgenössischen uigurischen Verhältnisse widerspiegeln ${ }^{43}$.

${ }^{38}$ S. Anm. 35.

${ }^{39}$ Vgl. K. Röhrborn: Uigurisches Wörterbuch, Lief. 1, 1977, 43 b- 45 a.

41) Vgl. op. cit., Lief. 4, 1988, s. v. atl(2)g (II), 272 b-273 b.

${ }^{41}$ Vgl. Laut, Der frühe türkische Buddhismus (s. Anm. 2), 143148.

${ }^{42}$ Es sollen hier nicht die Fälle verschwiegen werden, die nıcht so eındeutıg wie die erwàhnten Beıspıele auf alternative Lesarten hinweisen und für die ich nicht meıne Hand ins Feuer legen móchte: Z. 314: Hier ist

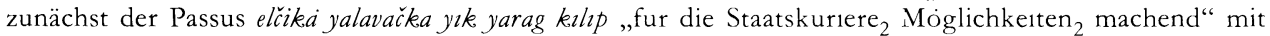
kleinen Kreisen unterlegt; superskribiert ist ič taš kišilarig temadin „ohne zwischen eigenen und fremden Leuten zu unterscheiden (?)“. In der gleichen Zeile ist weiterhin, nach dem Wort intkisin „das Passende

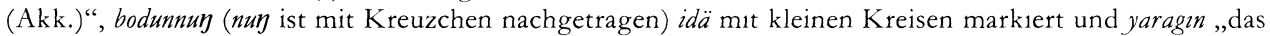
Passende (Akk.)" superskribiert. Wenn wir annehmen, die untürkische Wortstellung des zwerten Berspiels sei auf die Alliteration zurückzuführen, so ließe sich bei einer Umstellung lesen: *bodunnuy ida intkisin turdač „die das Passende für das Volk wahren“. Eher scheint es mir, als sei der gesamte Passus dem Uiguren aus dem Leim gegangen, was bei einer ad hoc komponierten Predigt ja nicht auszuschließen ist. In Z. 315 sind im Satz

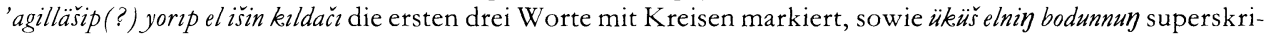

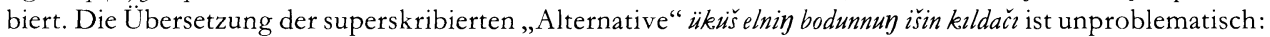
„die die Angelegenheiten vieler Völker 2 ausführen“, doch kann 'ägilläš̈ip(?) yorıtp el išin kelldač als „die die Angelegenheiten des unterdrückt (<agil-?) lebenden Volkes ausführen" interpretiert werden?

${ }^{43}$ Vgl. K. Röhrborn: Türkische Philologie und Sprachwissenschaft in Japan, 18. In: Laut/Röhrborn (Hrsgg.): Der türkische Buddhismus (s. Anm. 35), 1-26. 


\section{Zusammenfassung}

Die Betrachtung von einer Auswahl von typischen Fehlern und ihren Korrekturen in alttürkischen Handschriften verschiedenen Alters zeigt: In den alten, sorgfältig geschriebenen Manuskripten, die zum größten Teil im Auftrage von Stiftern hergestellt wurden, werden wenig Korrekturzeichen verwendet. Die Schreiber versuchten, ihre Fehler im Interesse eines „sauberen“ Manuskriptes so unauffällig wie möglich zu korrigieren. Bestimmte Fehlergruppen weisen darauf hin, daß diese Handschriften nach der Vorlage eines anderen Manuskriptes und nicht nach Diktat geschrieben wurden. Die späten Manuskripte sind zumeist recht nachlässig geschrieben und weisen mehrere Korrekturzeichen auf, deren Funktion bei den verschiedenen Schreibern oft die gleiche ist. Ein Zeichen in Avadāna-Texten zeigt jedoch keine Emendationen, sondern alternative Lesarten an. Die späten Handschriften scheinen zum großen Teil nicht mehr im Auftrage von Stiftern hergestellt zu sein, sondern sind innerklösterliche Erzeugnisse für die mönchische Praxis, oft auch für die Predigt vor Laien. 


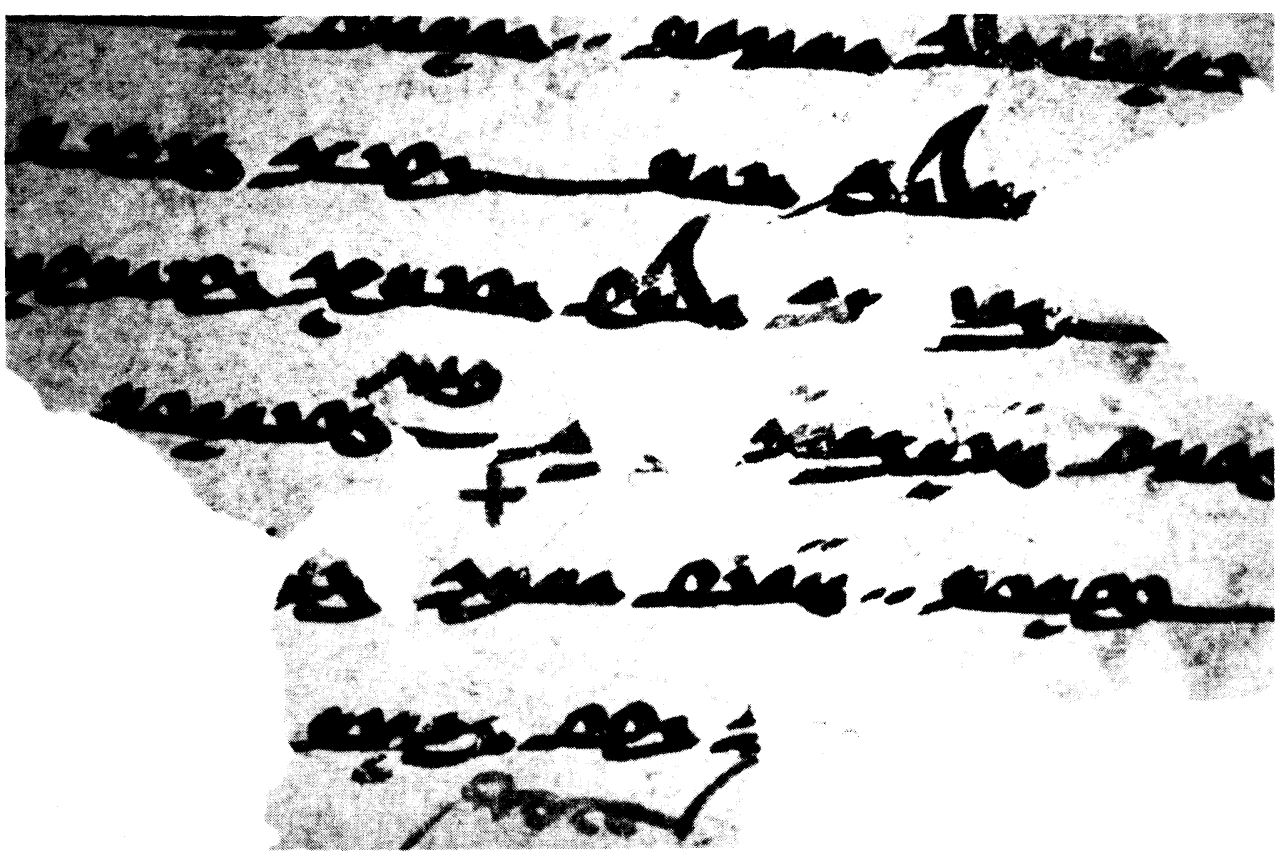

Abb. 1

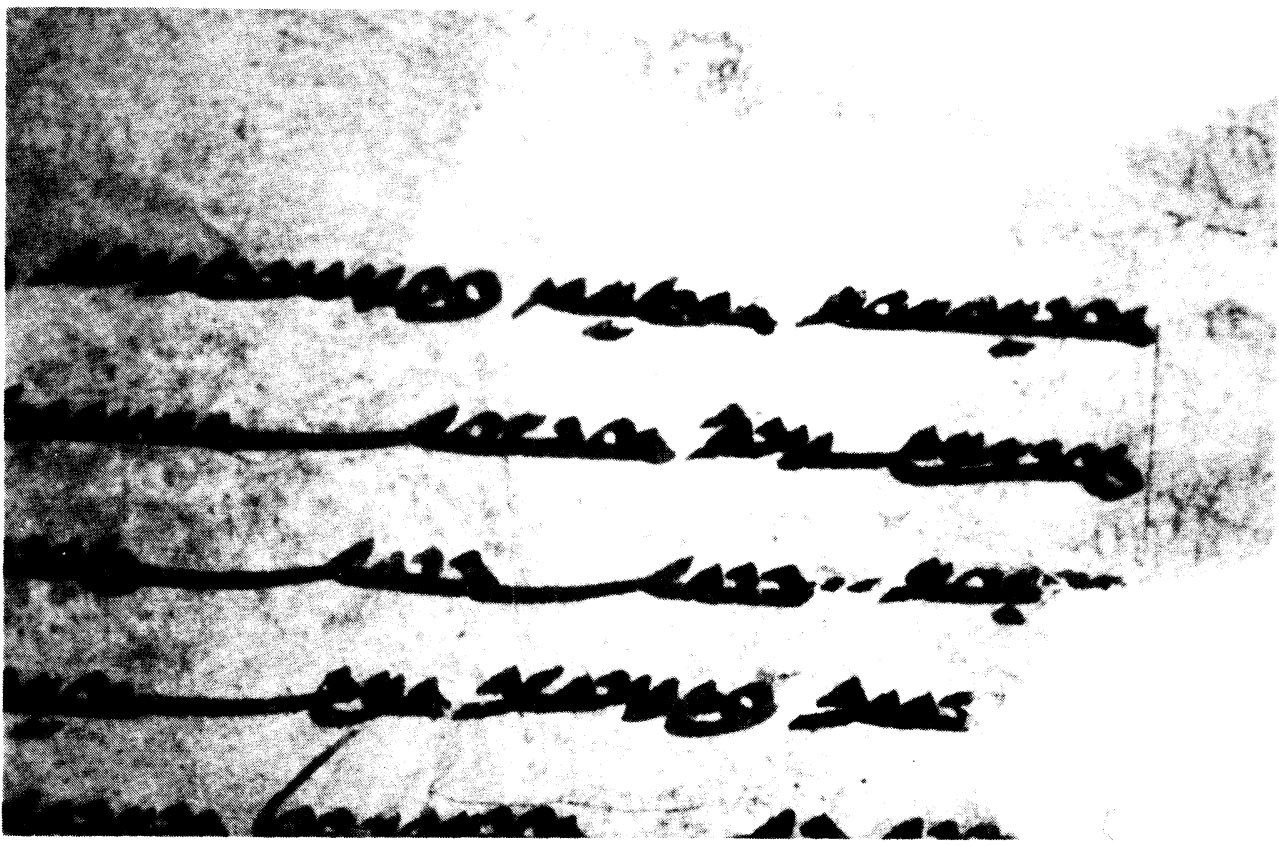

Abb. 2 


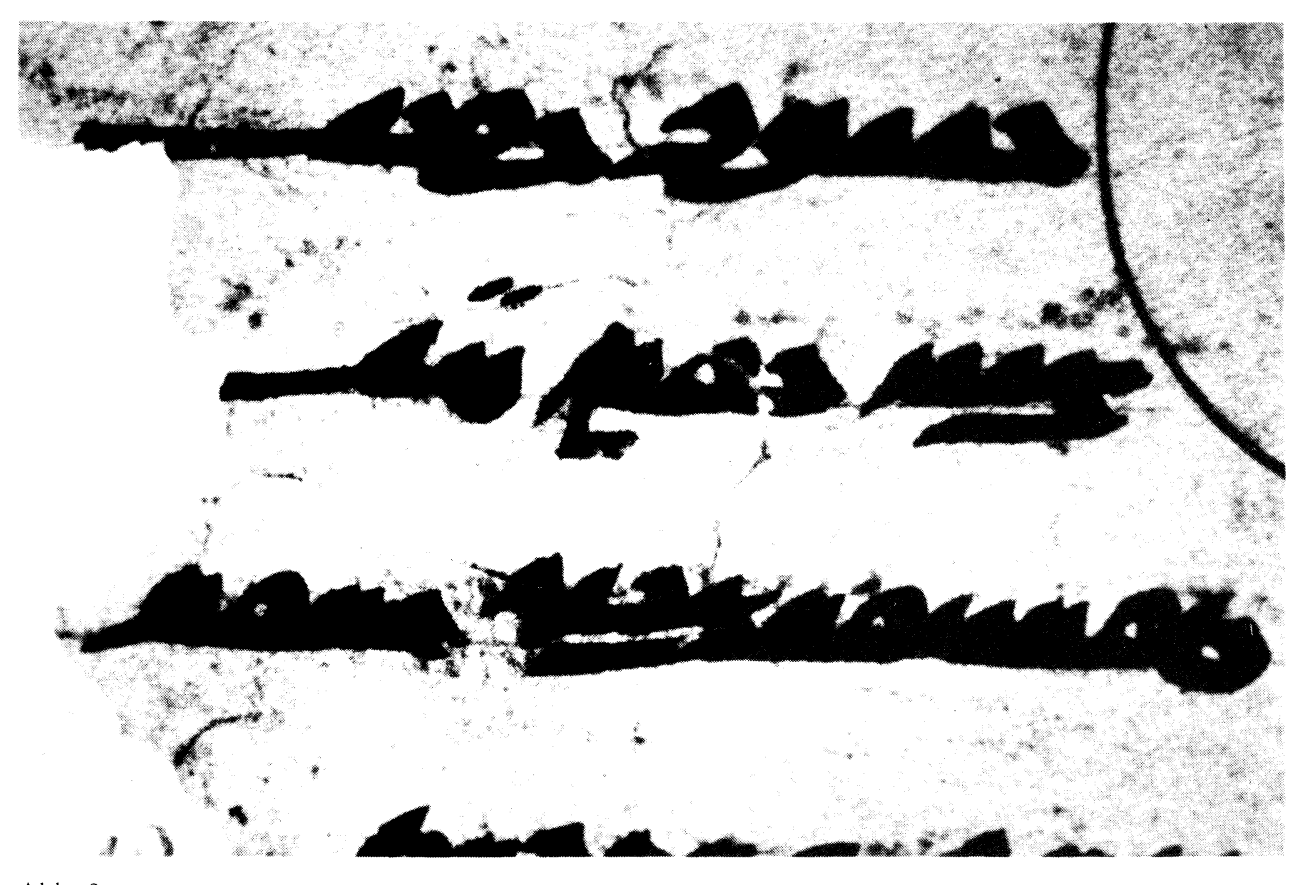
Abb. 3

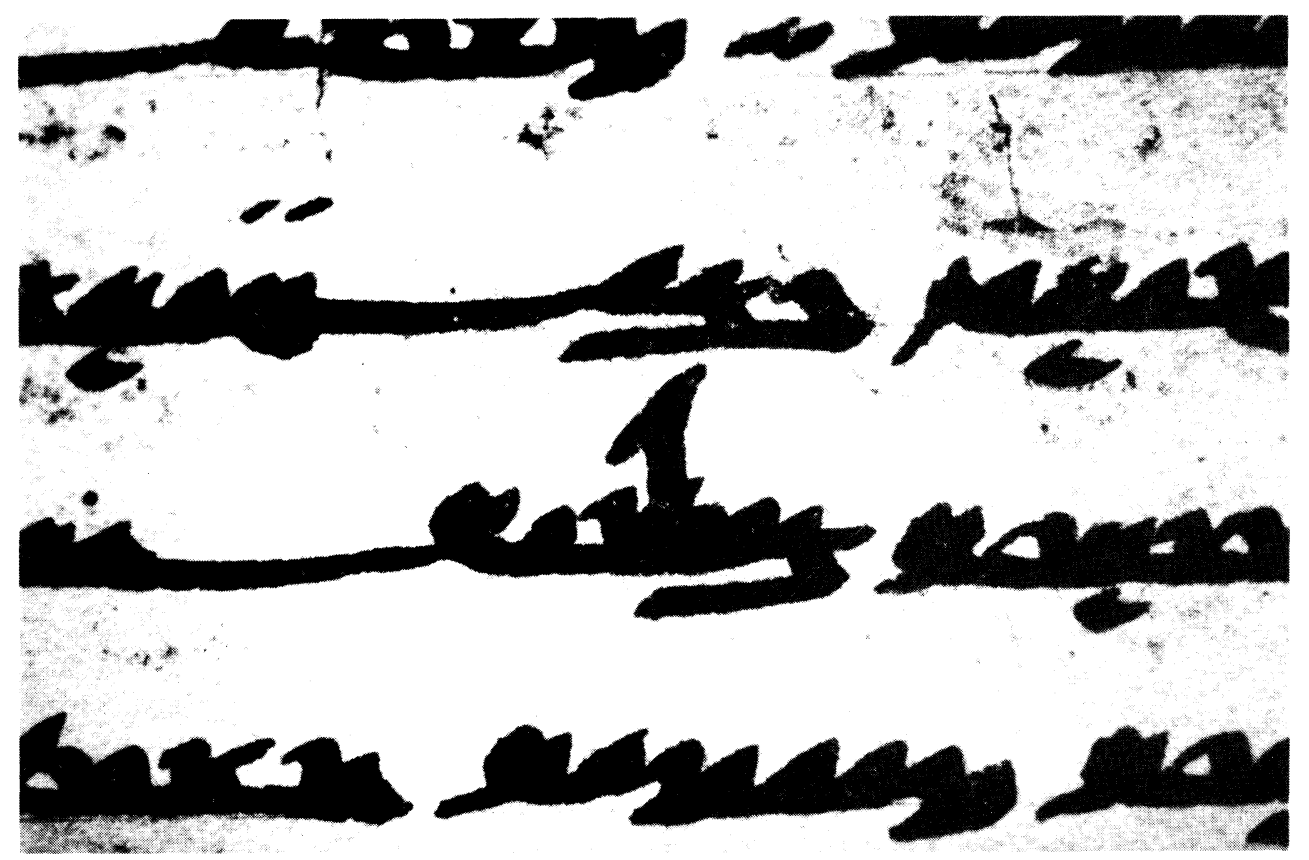
Abb. 4 


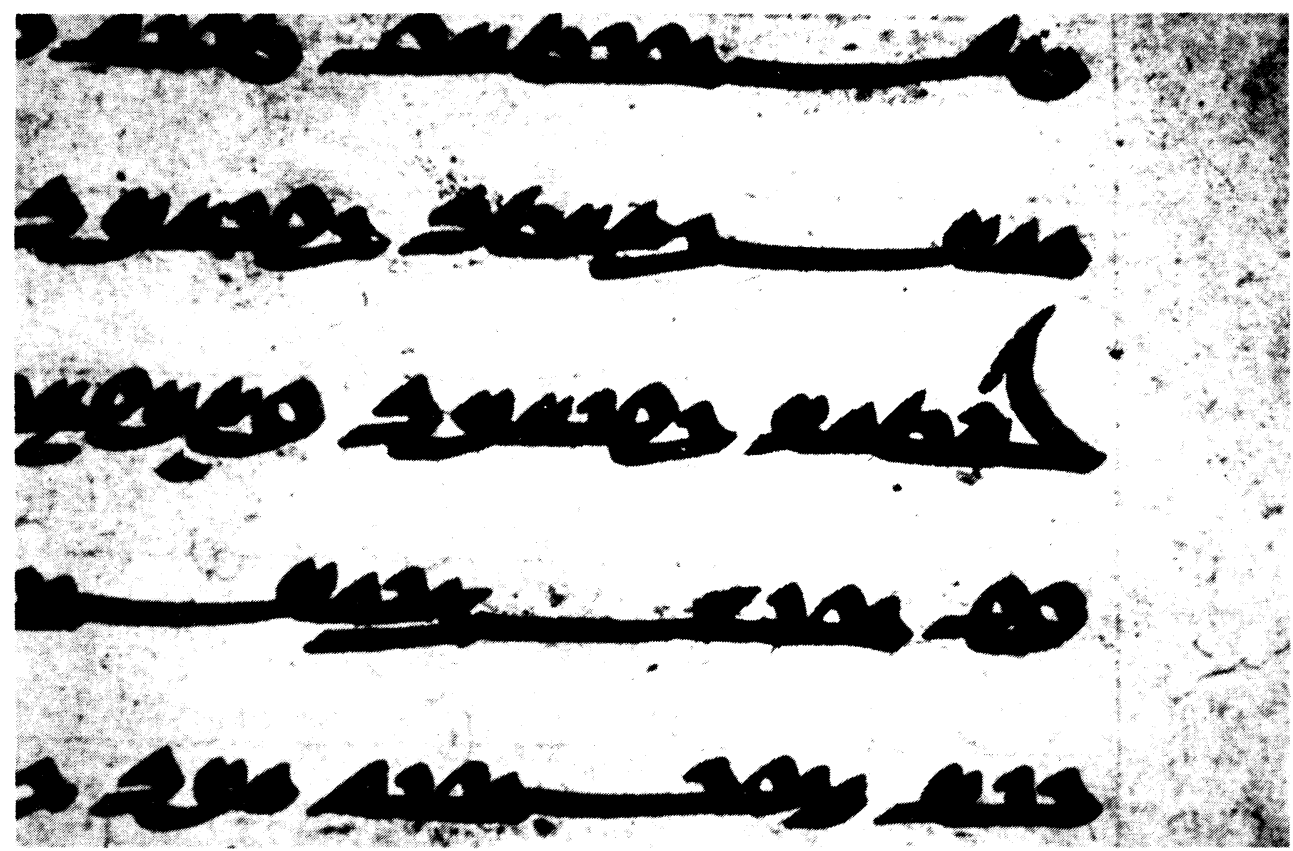
Abb. 5

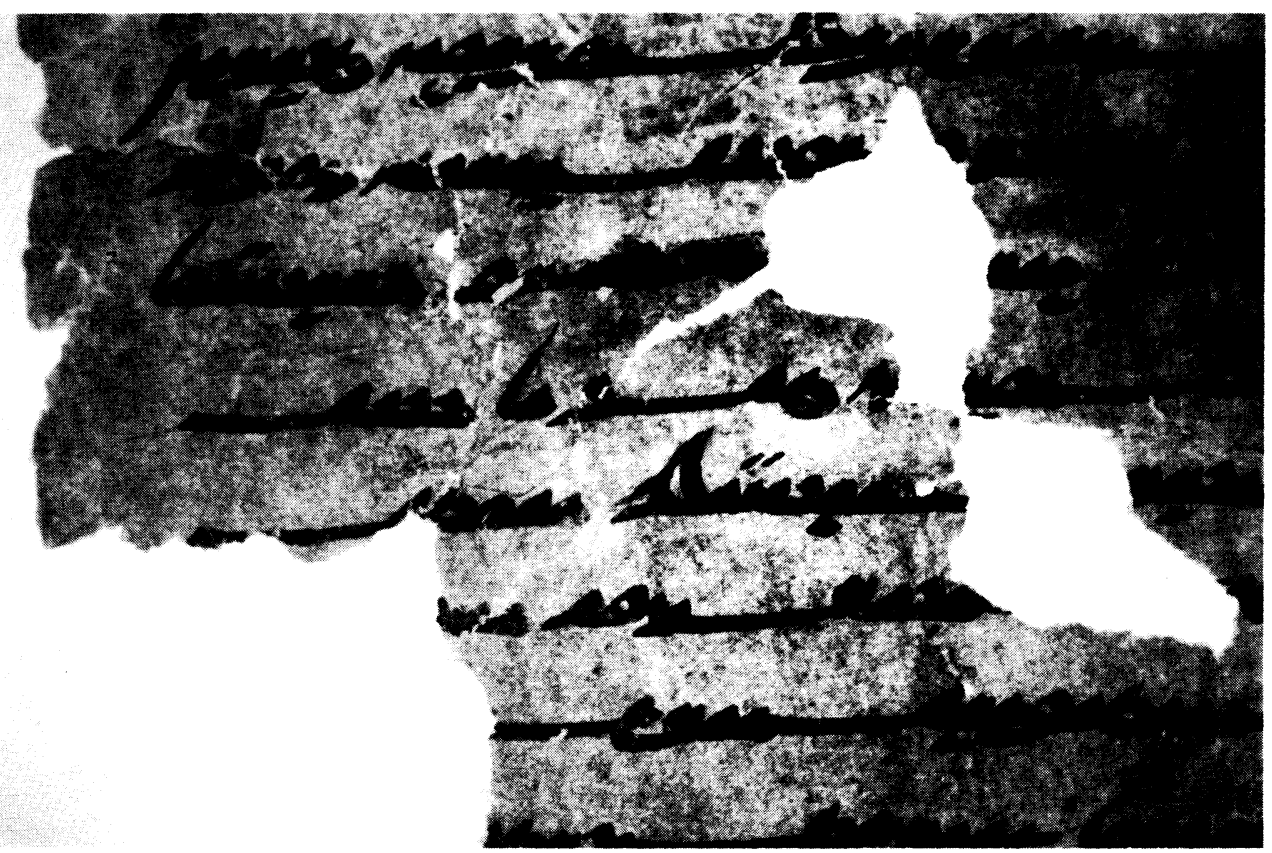

Abb. 6 


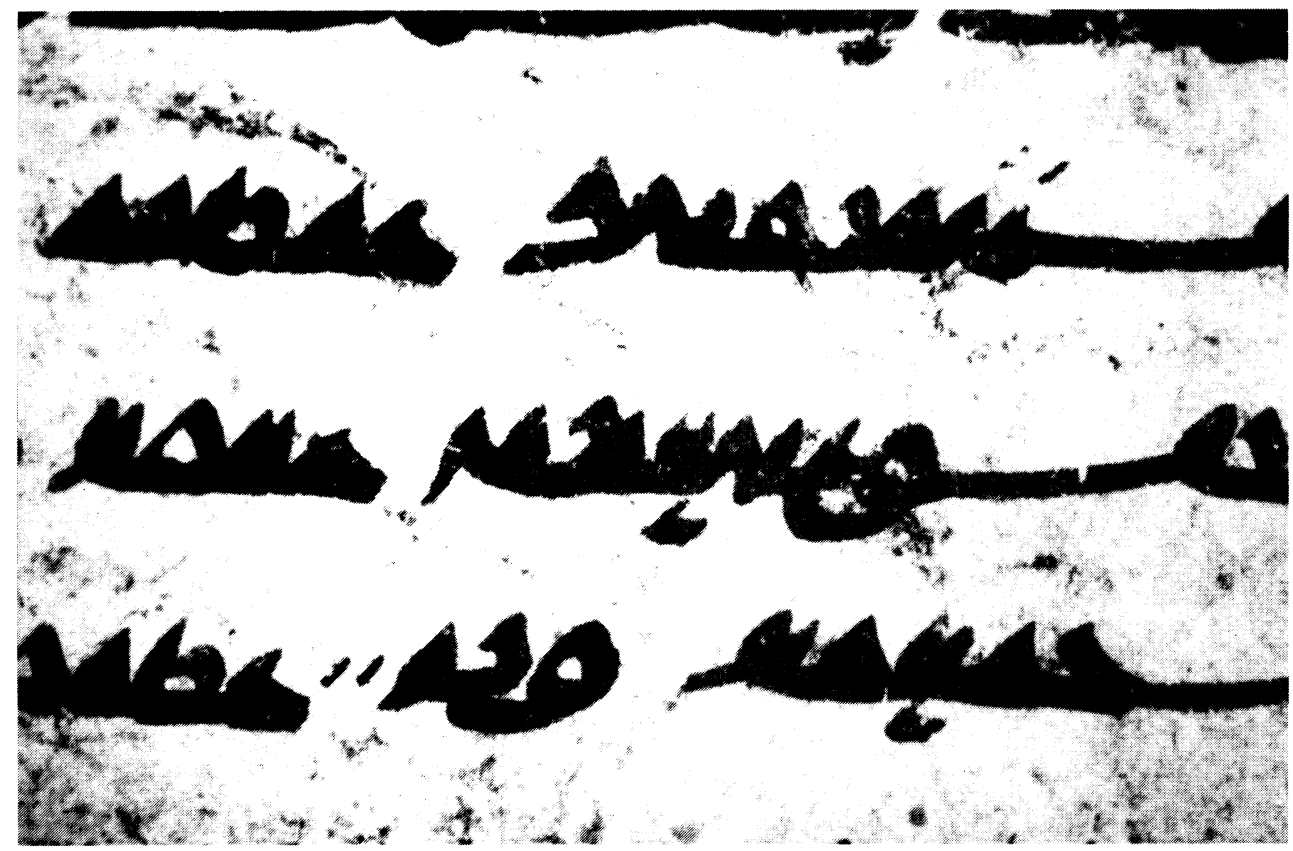
Abb. 7
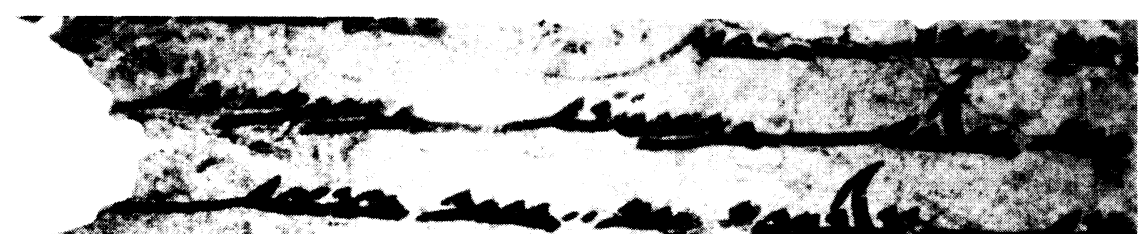

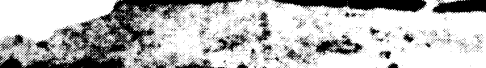

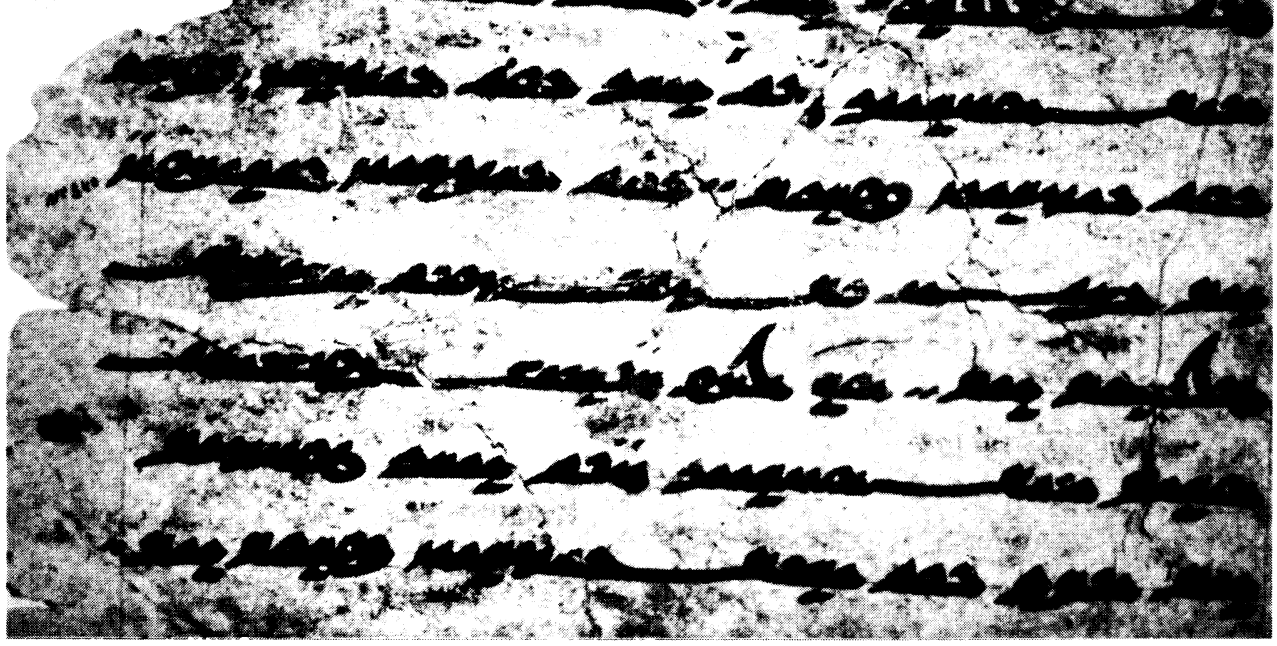

Abb. 8 


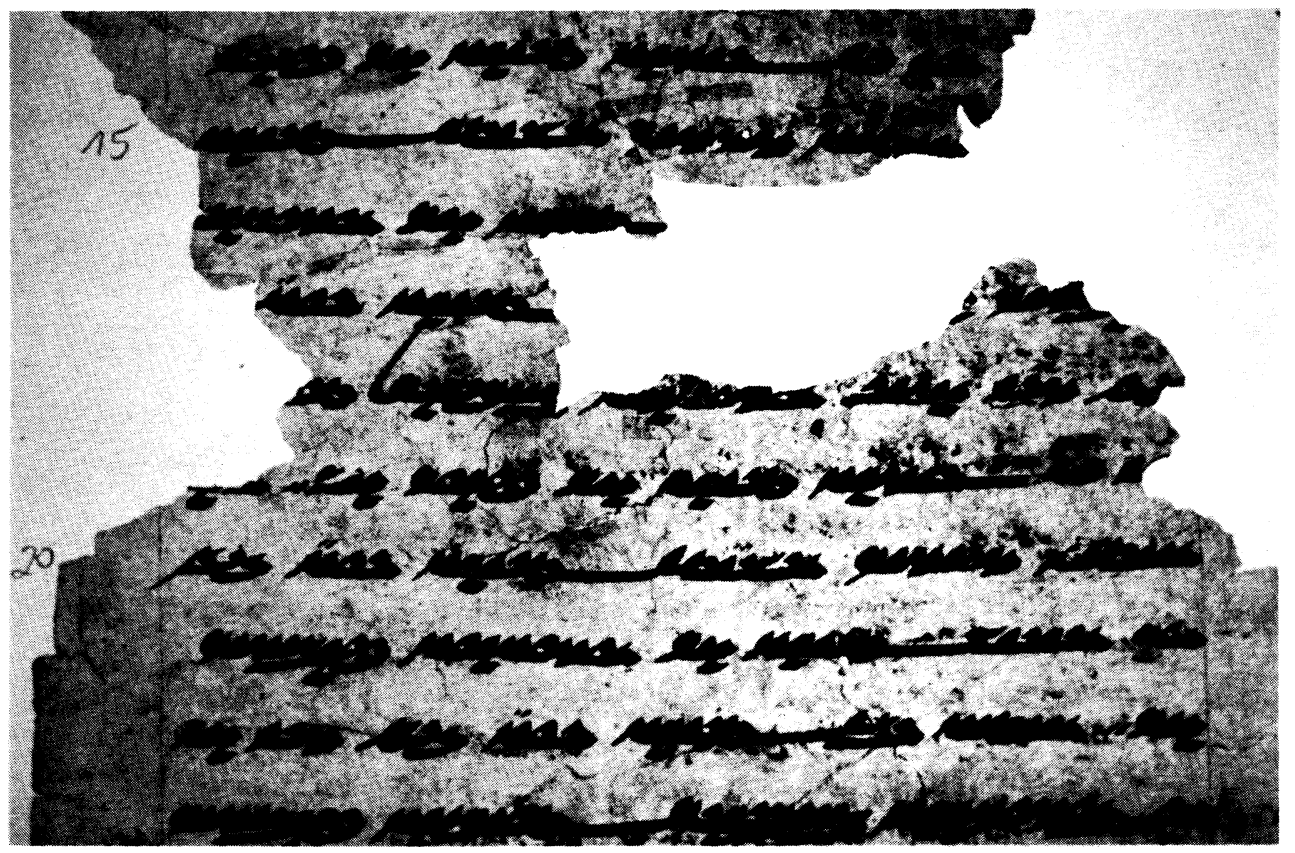

Abb. 9

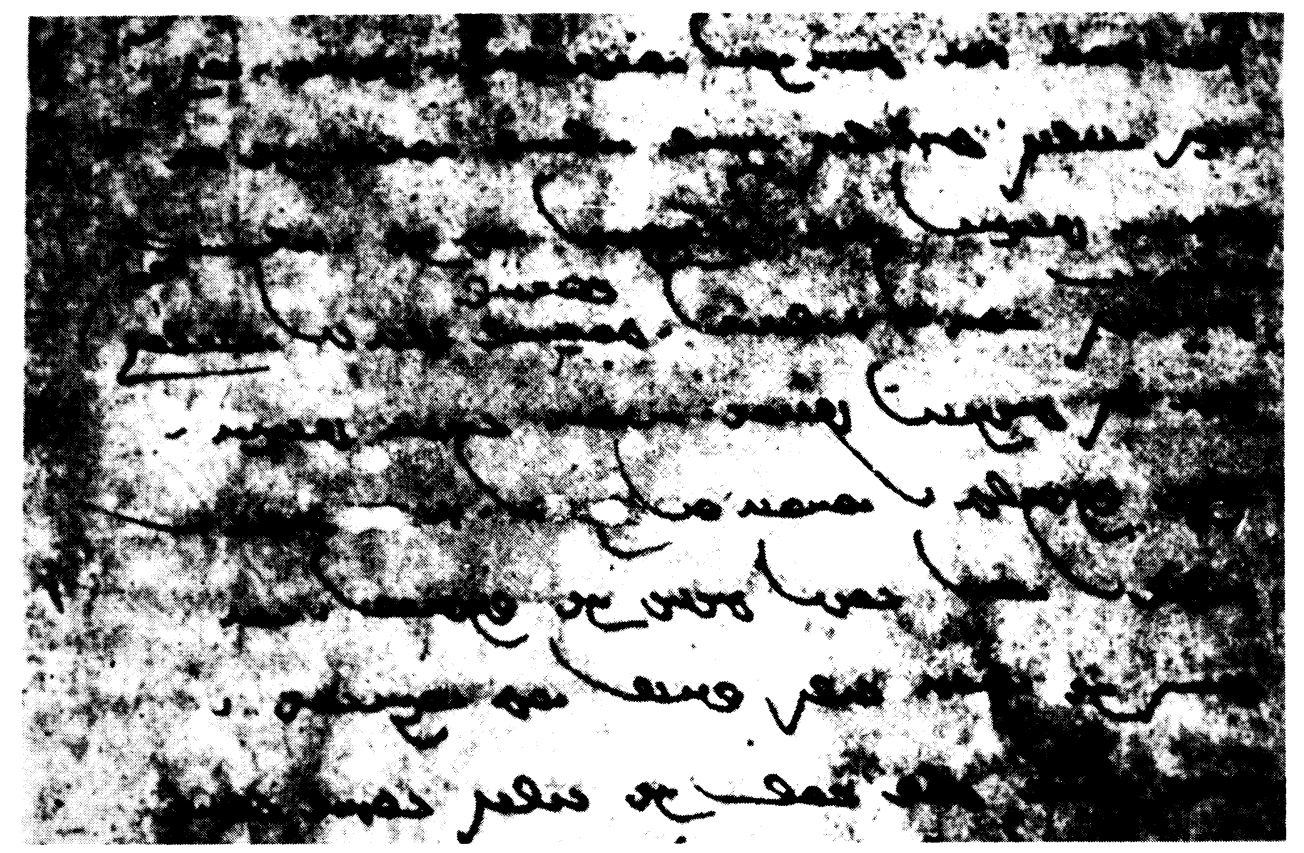

Abb. 10 


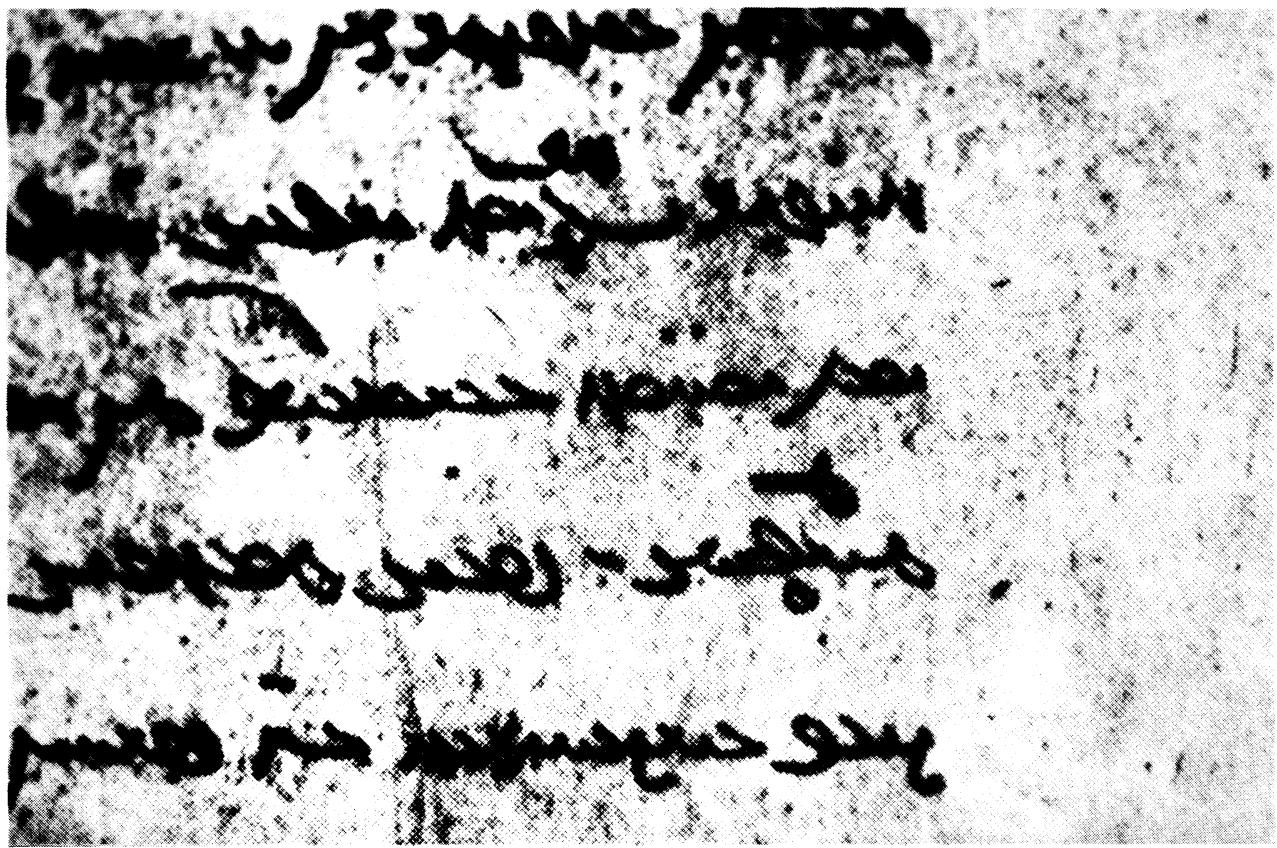

Abb. 11

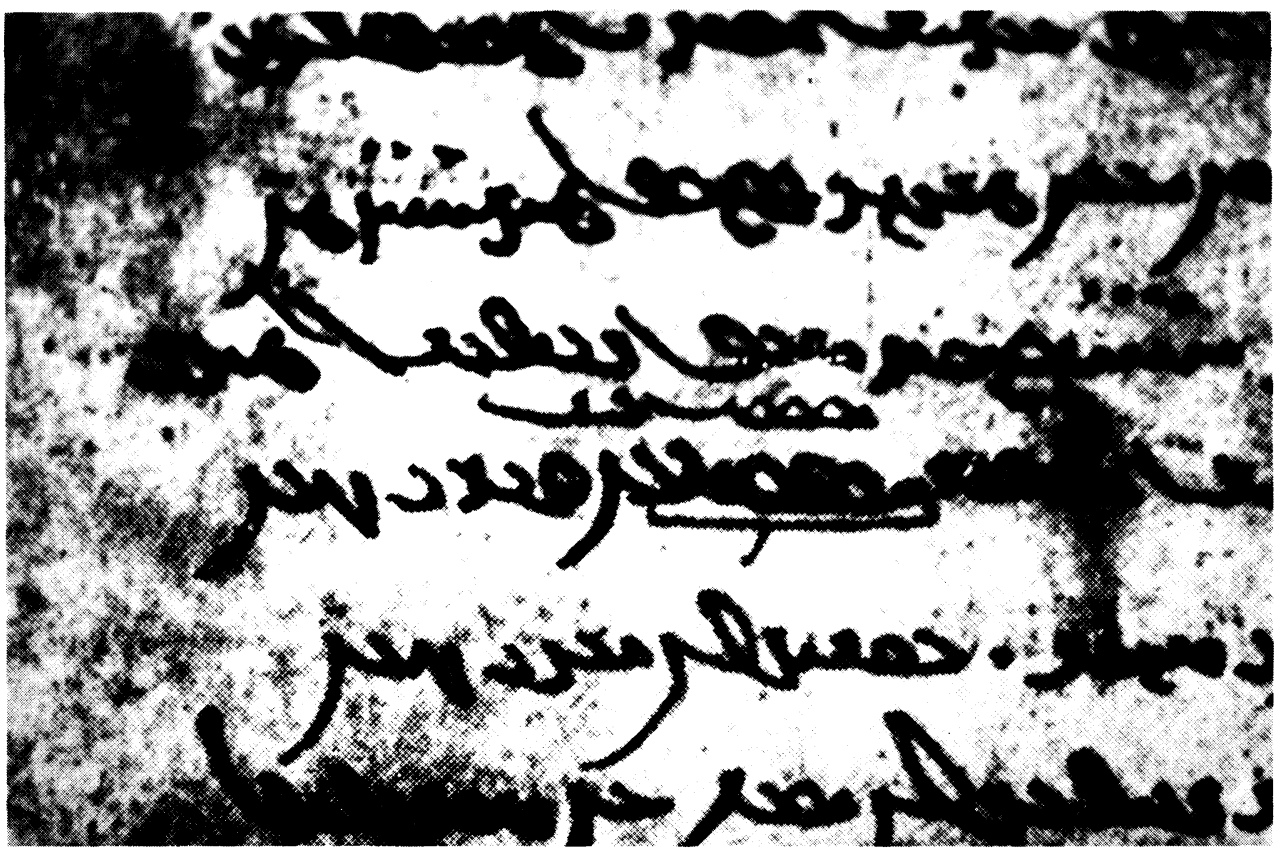

Abb. 12 


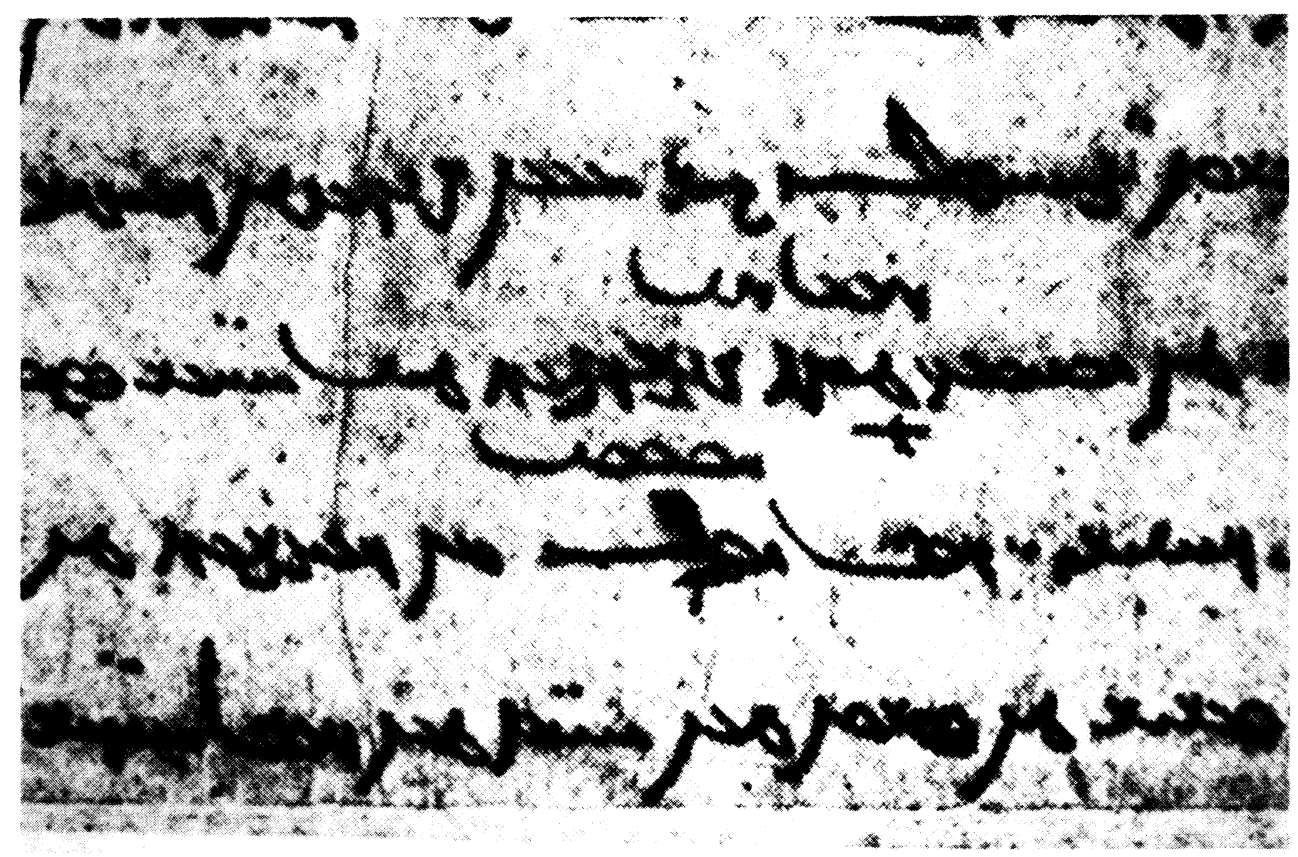

Abb. 13

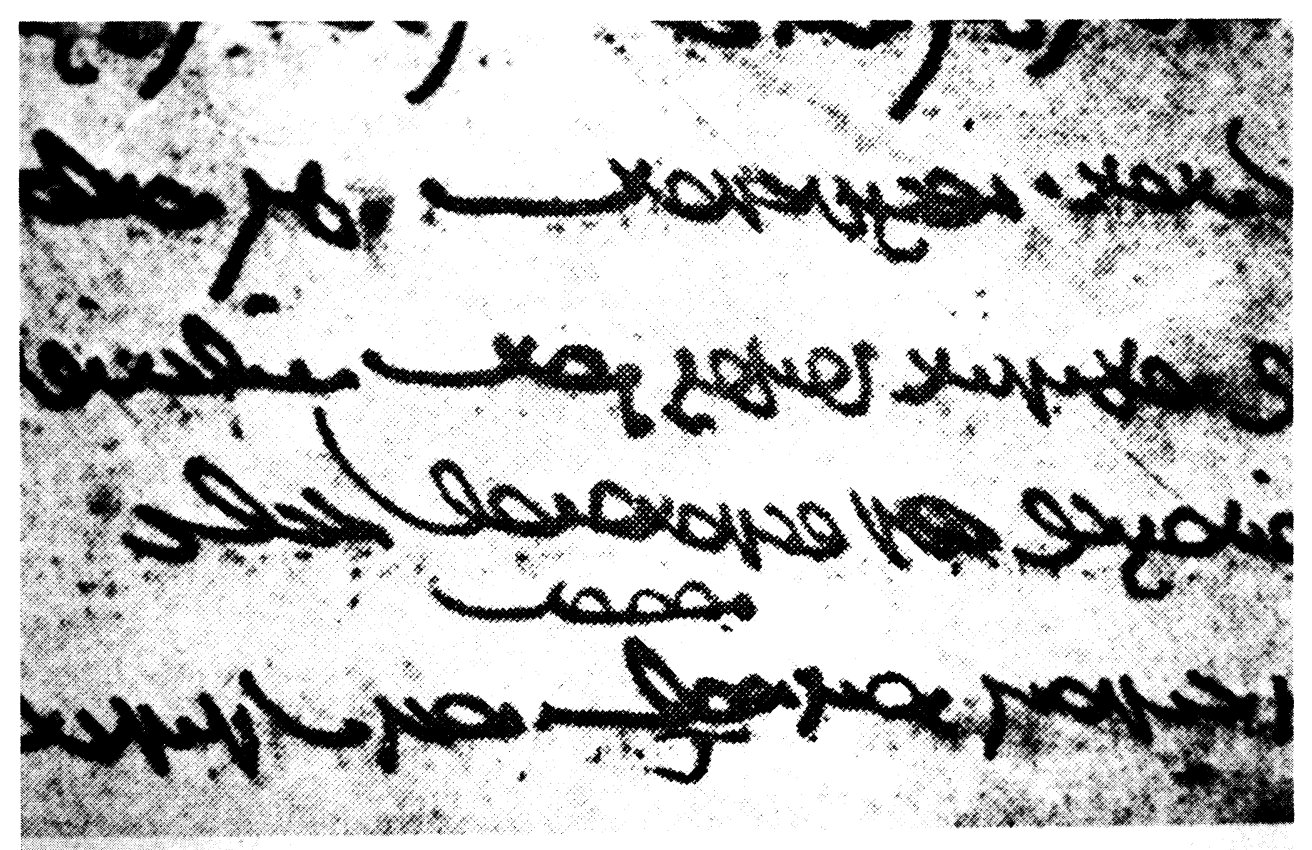




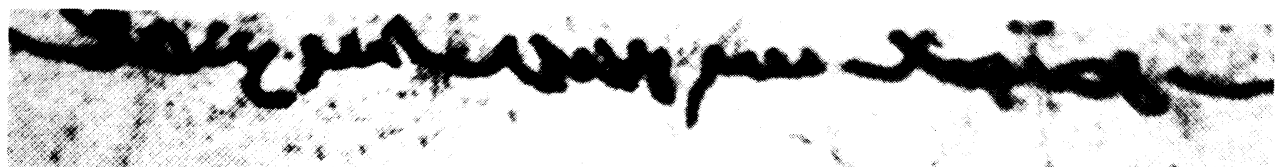

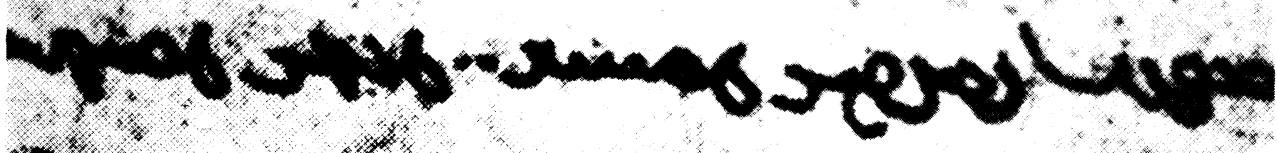
s.…

$F_{0}$

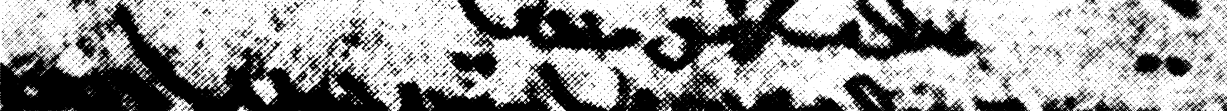
年,

(a) Abb. 15

1. سي

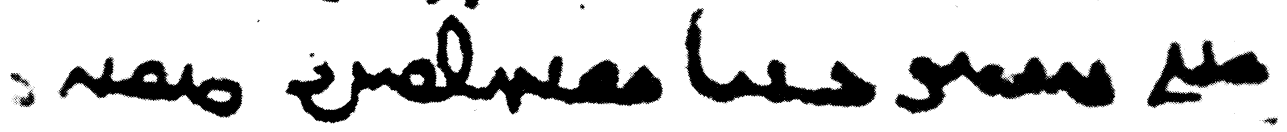

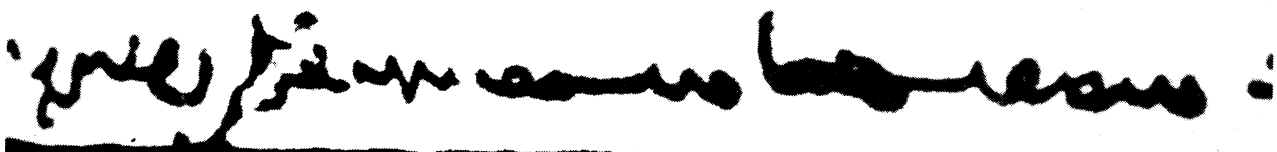

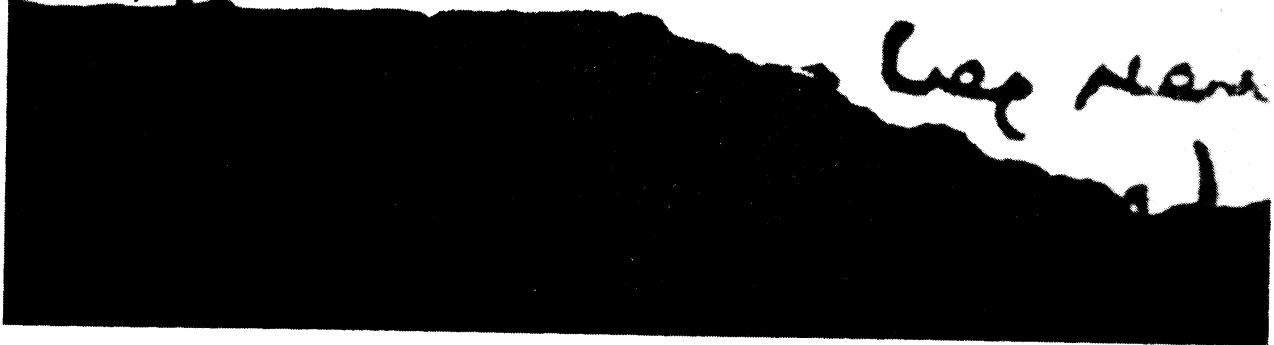




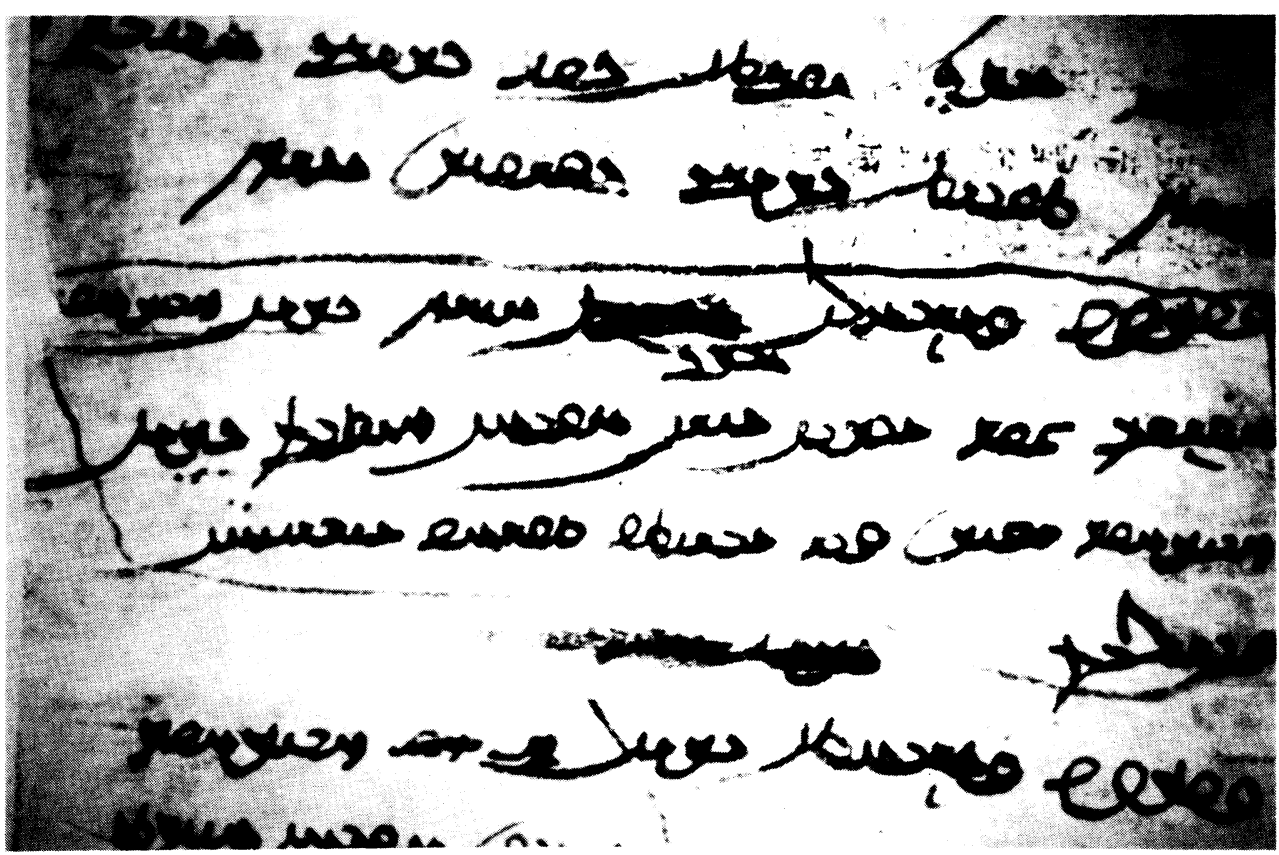

Abb. 17

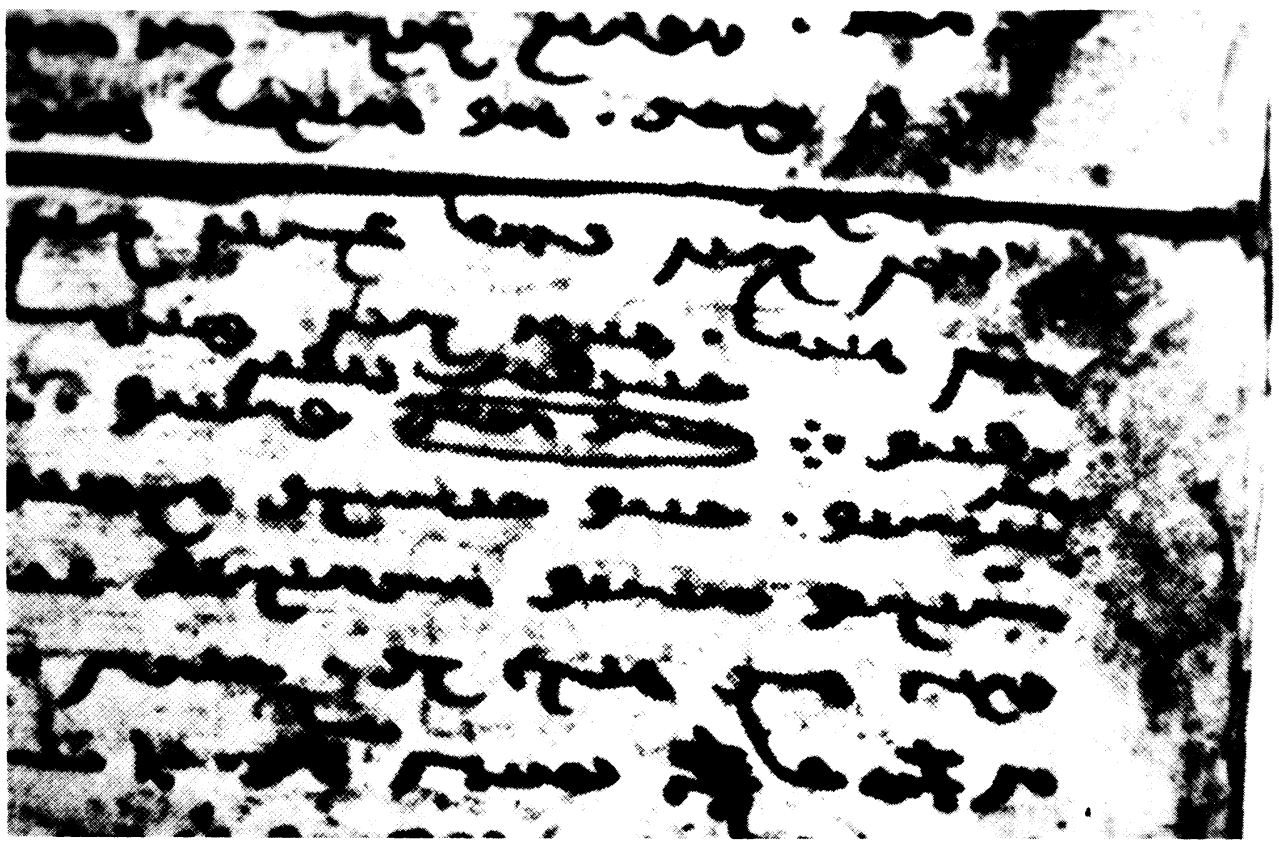




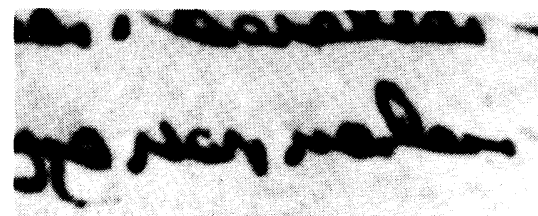

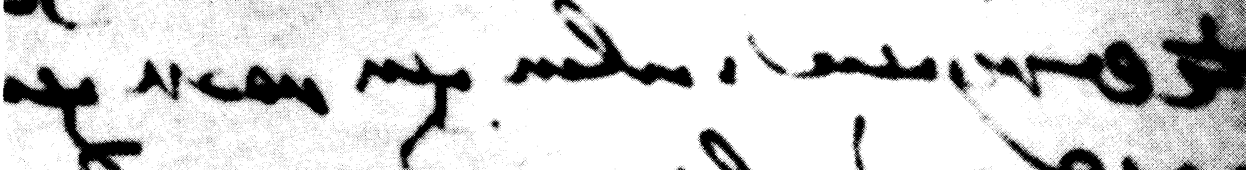

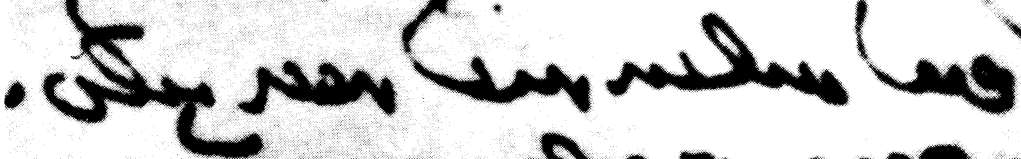

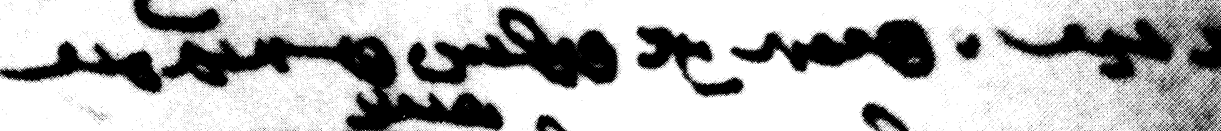
is 1 -

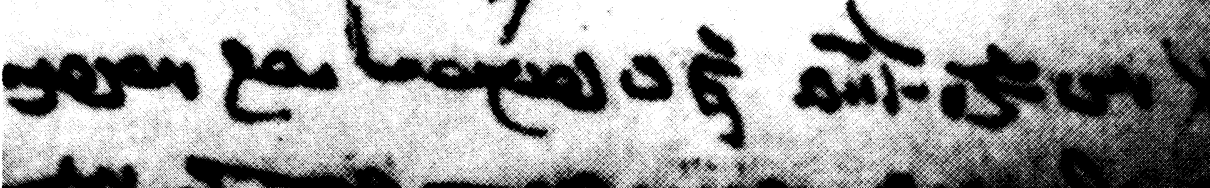

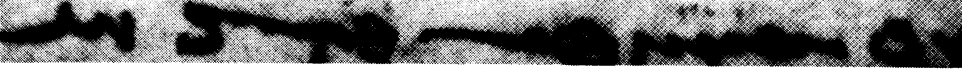

Abb. 19

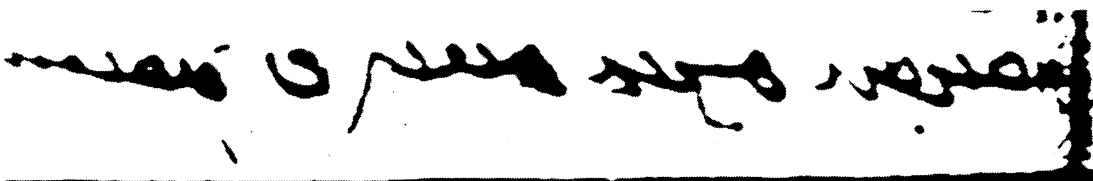

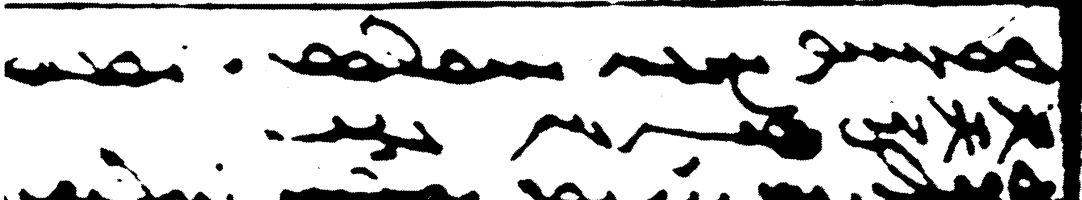
( -

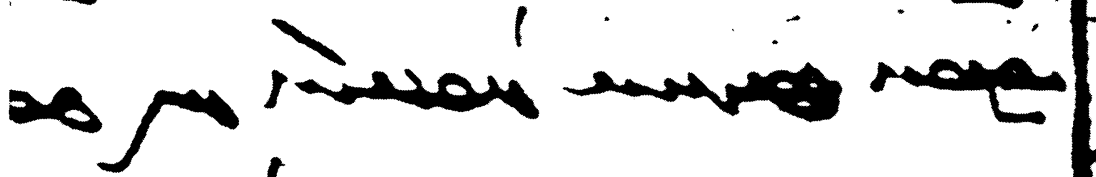

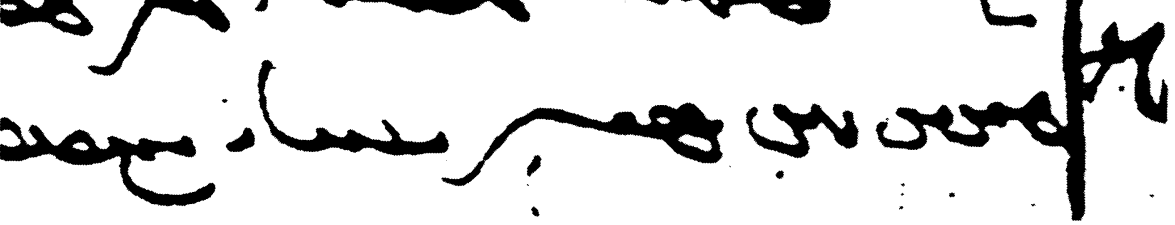

Abb. 20 


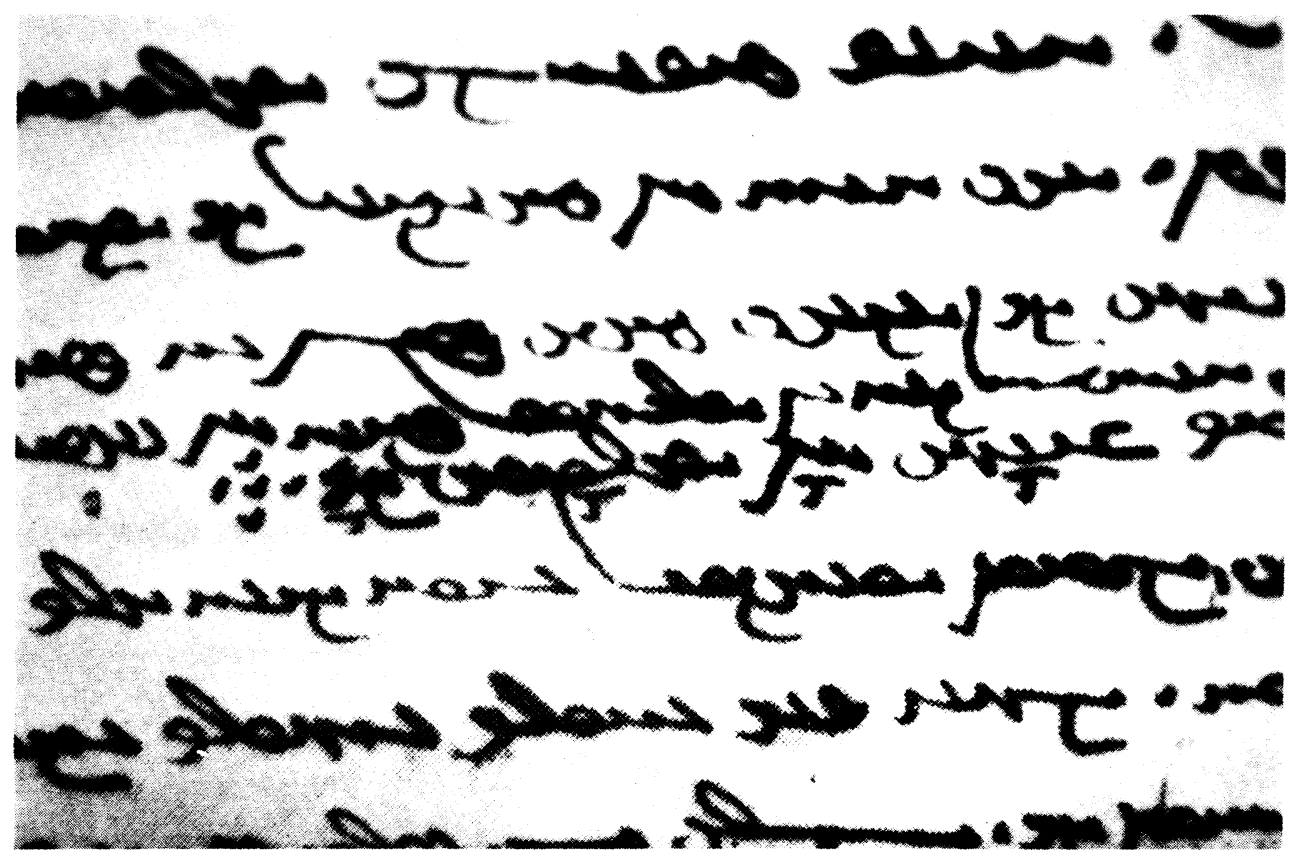

Abb. 21

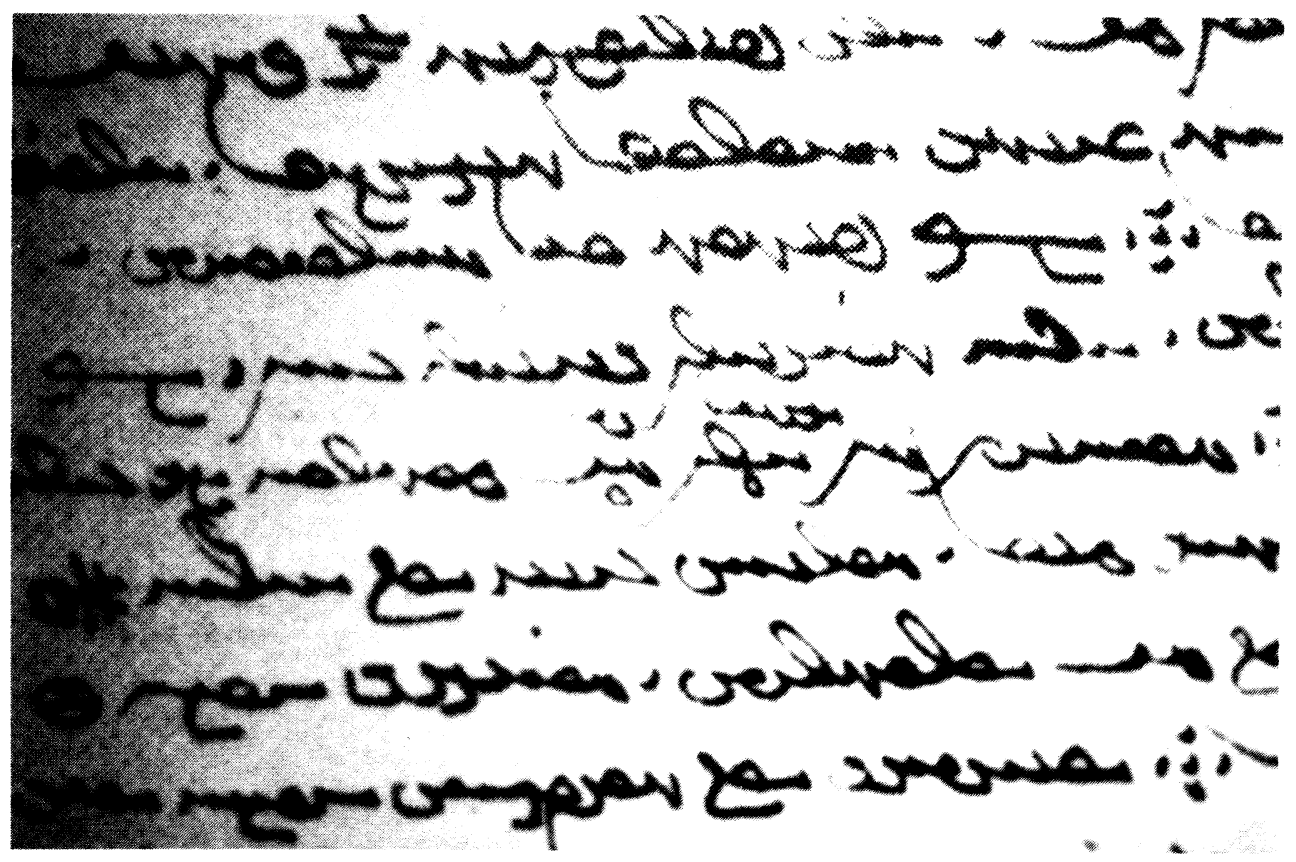




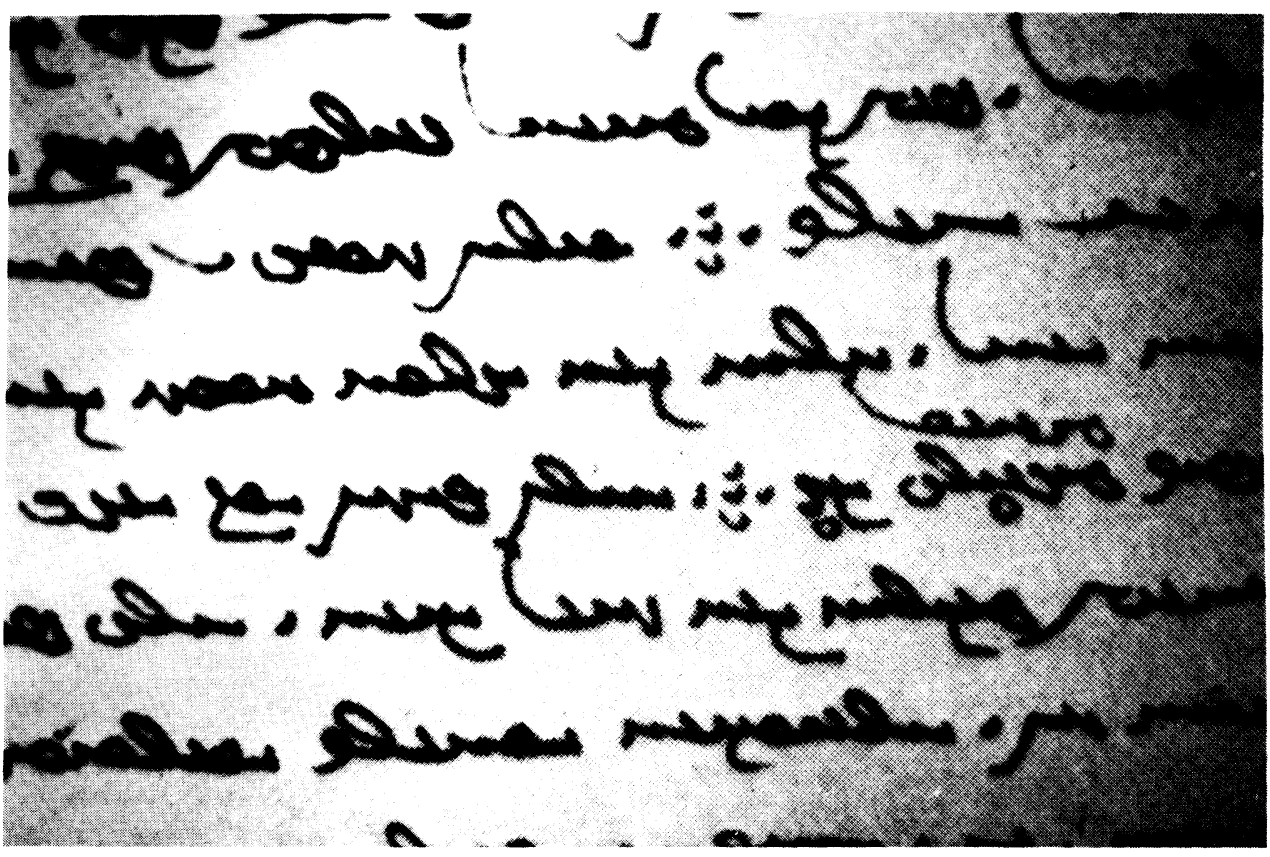

Abb. 23

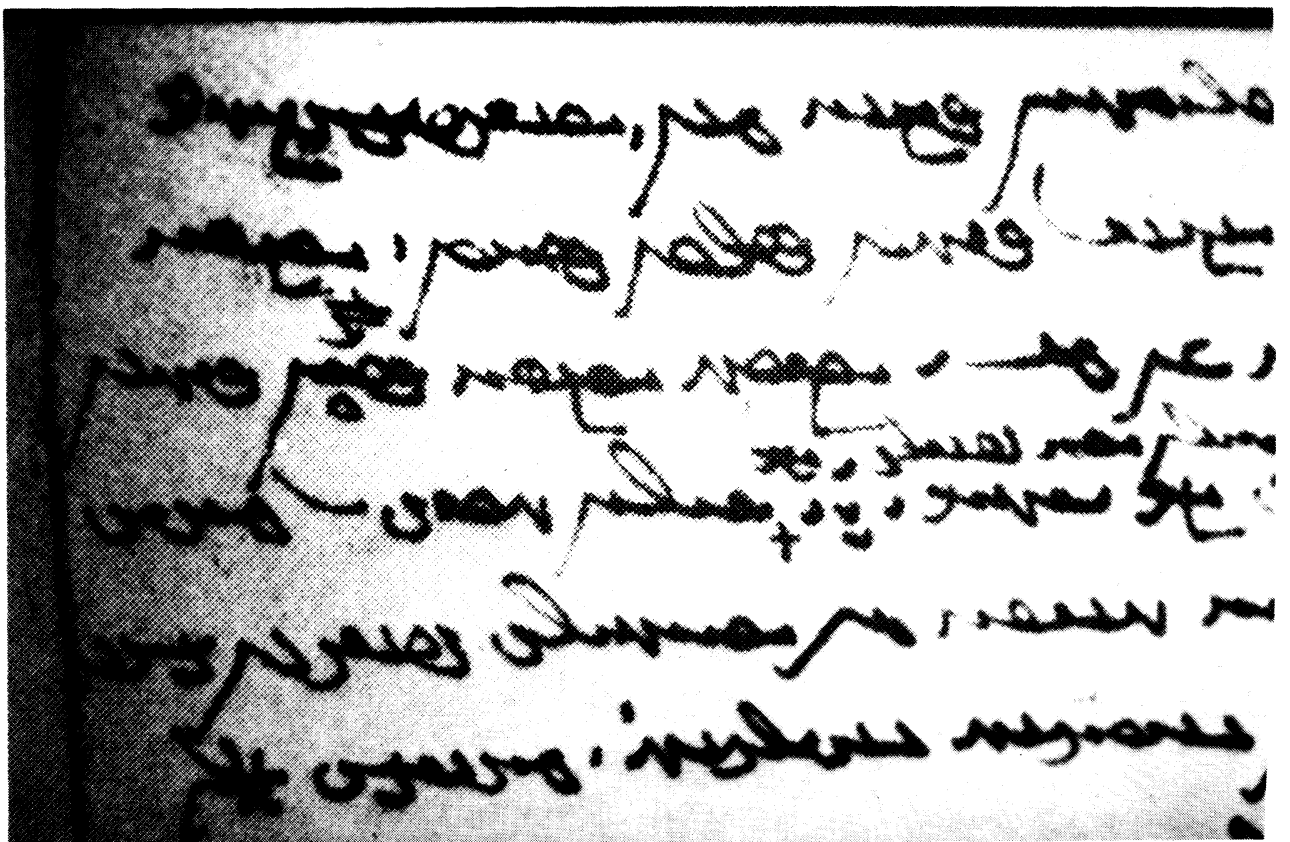

Abb. 24 


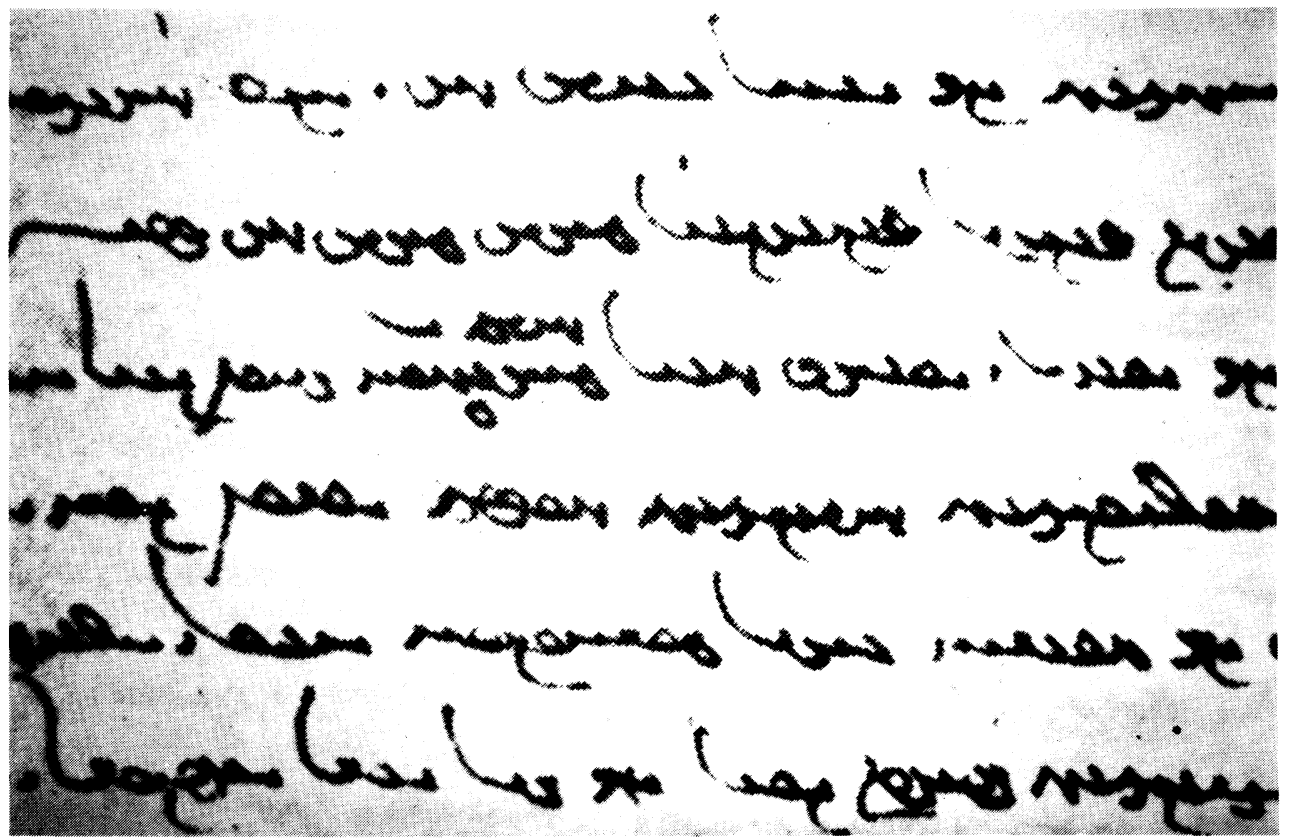

Abb. 25

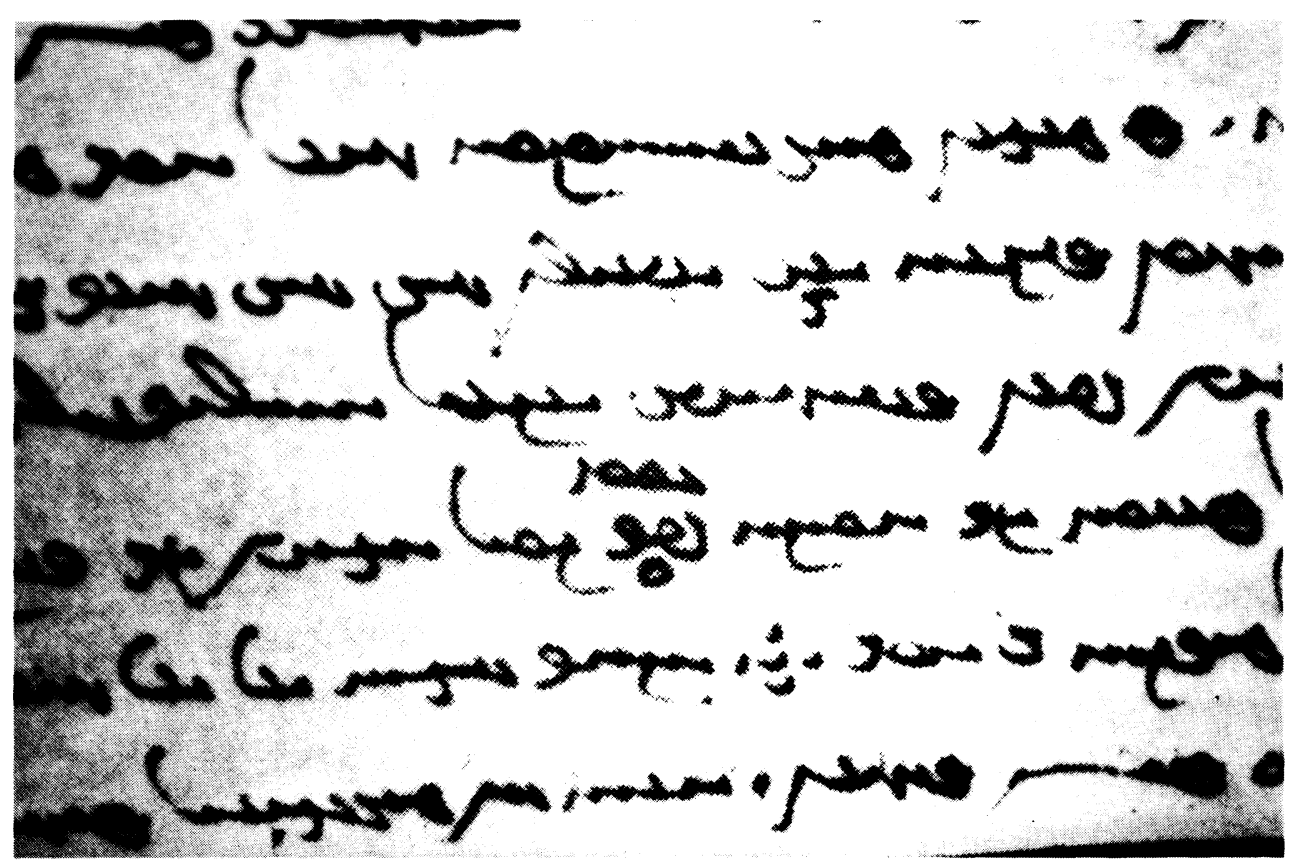

Abb. 26 